Supporting Information

\title{
Chiral Erbium(III) Complexes: Single-Molecule Magnet Behavior, Chirality, and Nuclearity Control
}

Min Feng*,", Bang-Heng Lyu ${ }^{\dagger}$, Ming-Hui Wang ${ }^{\dagger}$, Wei-Wei Wu ${ }^{\dagger}$, Yan-Cong Chen ${ }^{\dagger}$, Guo-Zhang Huang ${ }^{\dagger}$, Wei-Quan Lin ${ }^{\ddagger}$, Si-Guo $\mathrm{Wu}^{\dagger}$, Jun-Liang Liu ${ }^{\dagger}$, and Ming-Liang Tong*,

'Key Laboratory of Bioinorganic and Synthetic Chemistry of the Ministry of Education, School of Chemistry, Sun Yat-Sen University, Guangzhou 510275, P. R. China

${ }^{*}$ School of Chemistry and Chemical Engineering, Guangzhou University, Guangzhou Higher

Education Mega Center No. 230 Wai Huan Xi Road, Guangzhou 510006, P. R. China

E-mail:fengm6@mail.sysu.edu.cn (M.Feng); tongml@mail.sysu.edu.cn (M.-L. Tong).

\section{Contents}

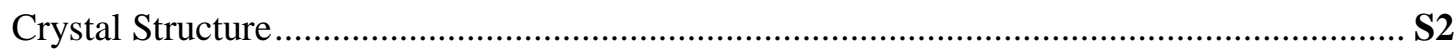

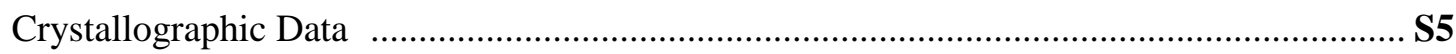

Geometry analysis, chirality analysis, selected bond lengths, angles ……………................ S6

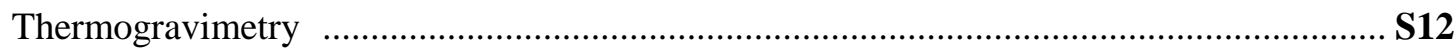

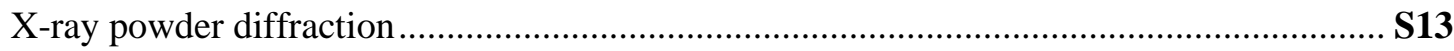

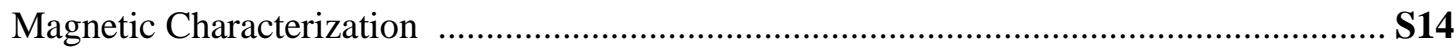

Ab Initio Calculations

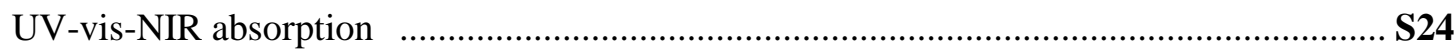




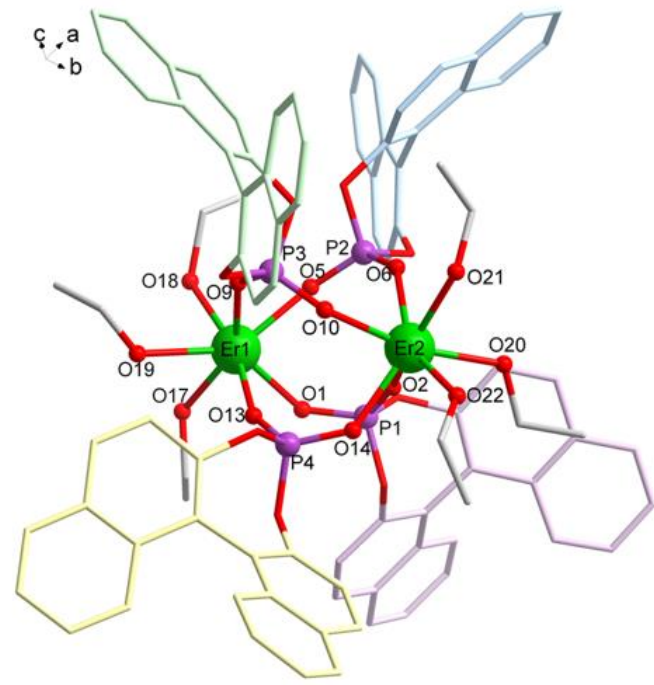

R-1

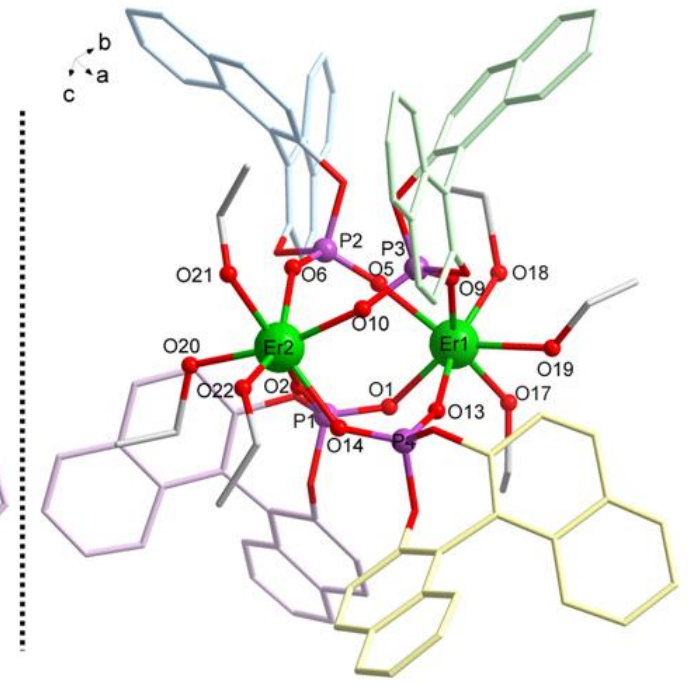

S-1

Figure S1. Molecular structure of $\boldsymbol{R}-\mathbf{1}$ and $\boldsymbol{S} \mathbf{- 1}$. Hydrogen atoms, counterions, and crystallization molecules have been omitted for clarity. Color code: Er, green; O, red; P, violet; C, light yellow, light pink, light blue, light green for different ligands, and gray for ethanol.

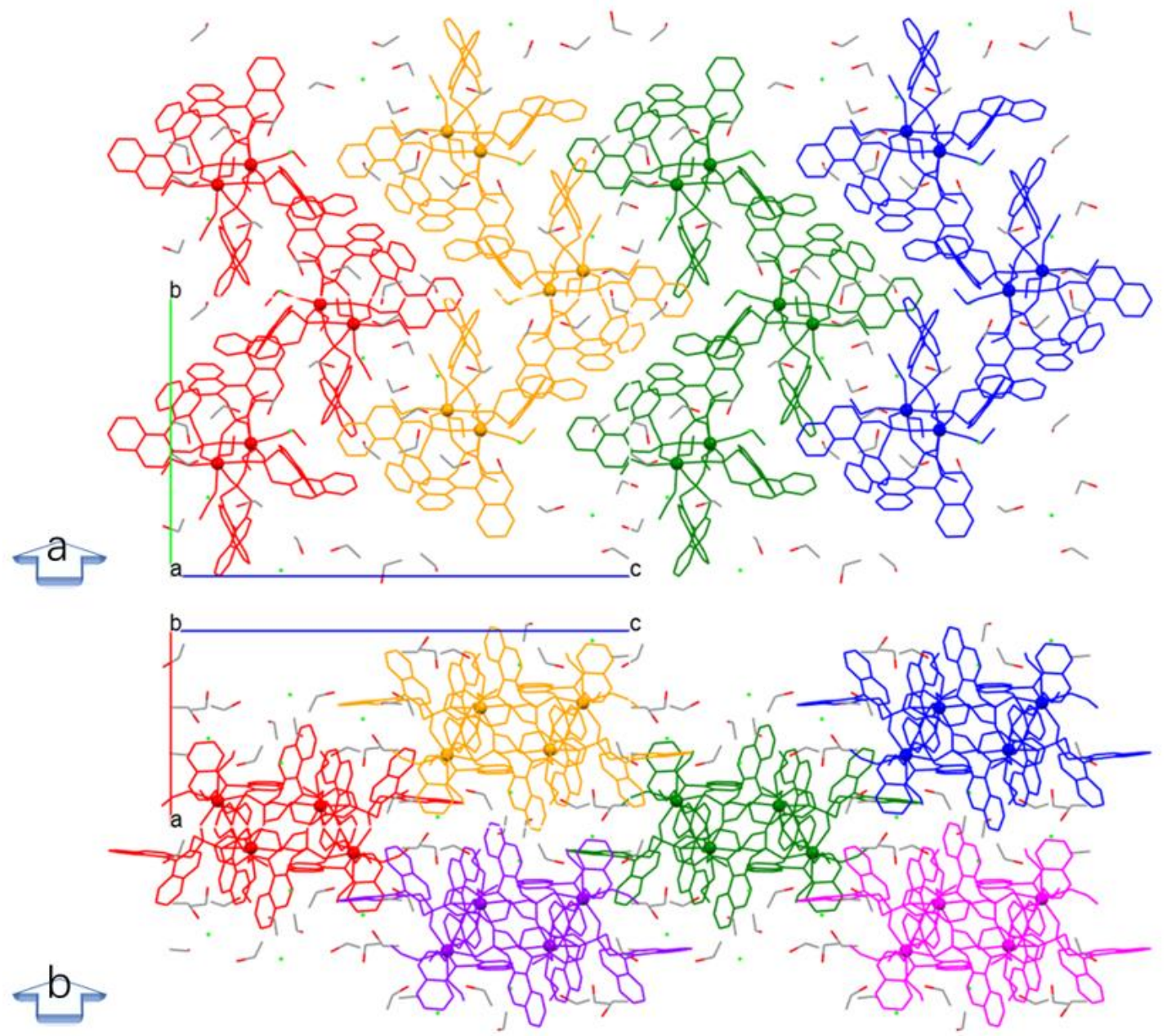

Figure S2. Crystal packing along a and b axes of $\boldsymbol{R}-\mathbf{1}$. 


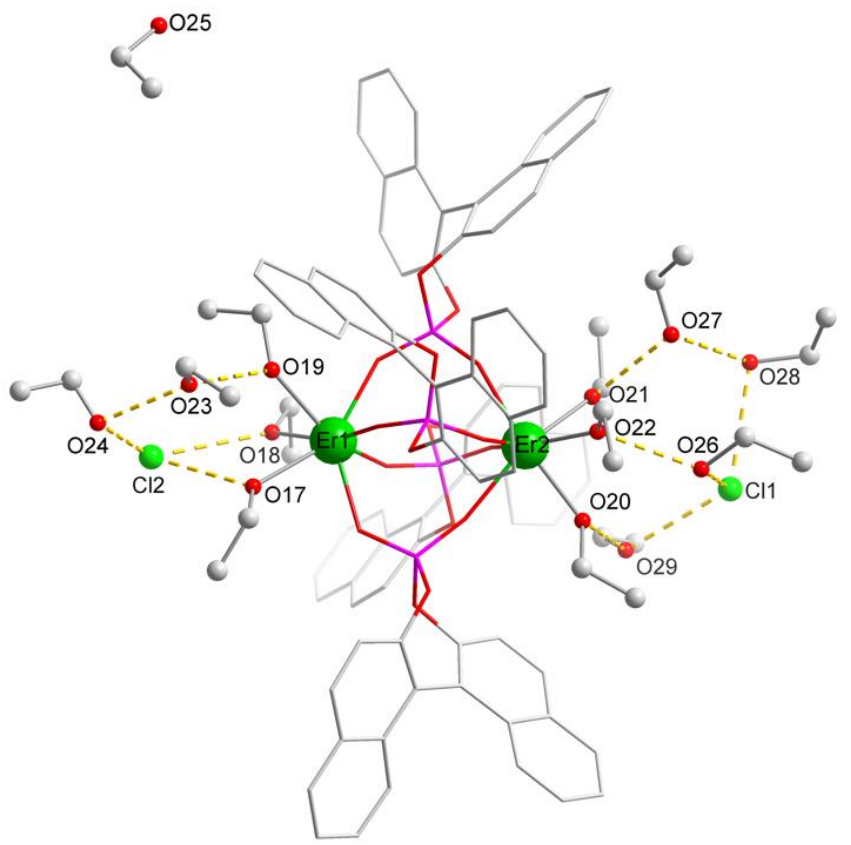

Figure S3. Hydrogen-bonding interactions between the coordinating ethanol molecules, the lattice ethanol molecules, and the chloride counterions. Color code: Er, green; $\mathrm{O}$, red; $\mathrm{P}$, violet; $\mathrm{Cl}$, bright green; C, gray.

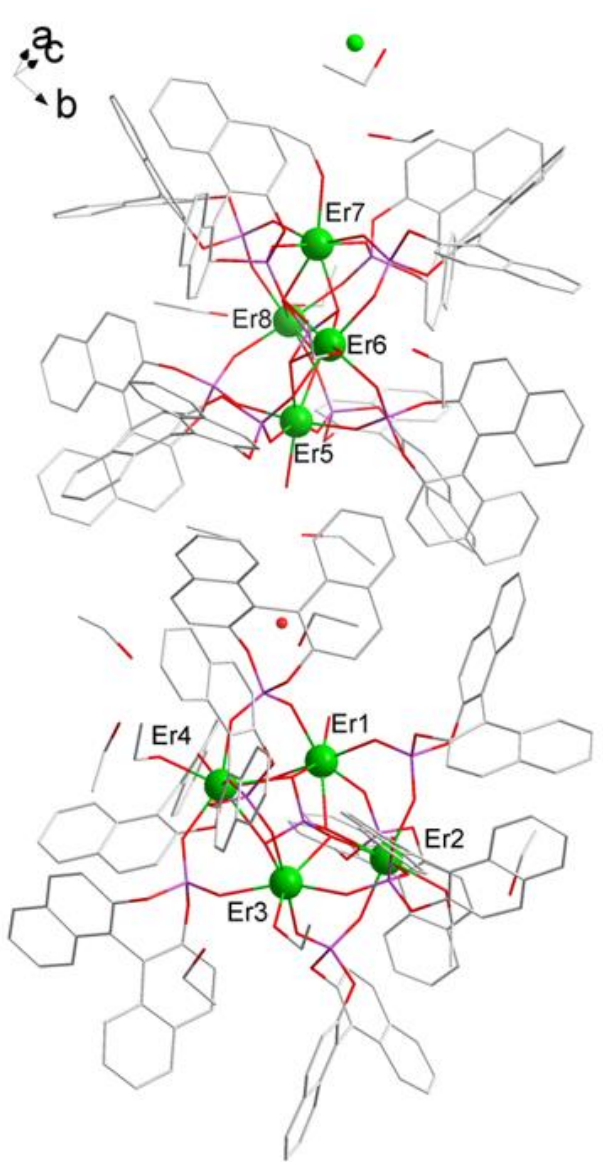

$\boldsymbol{R}-1$

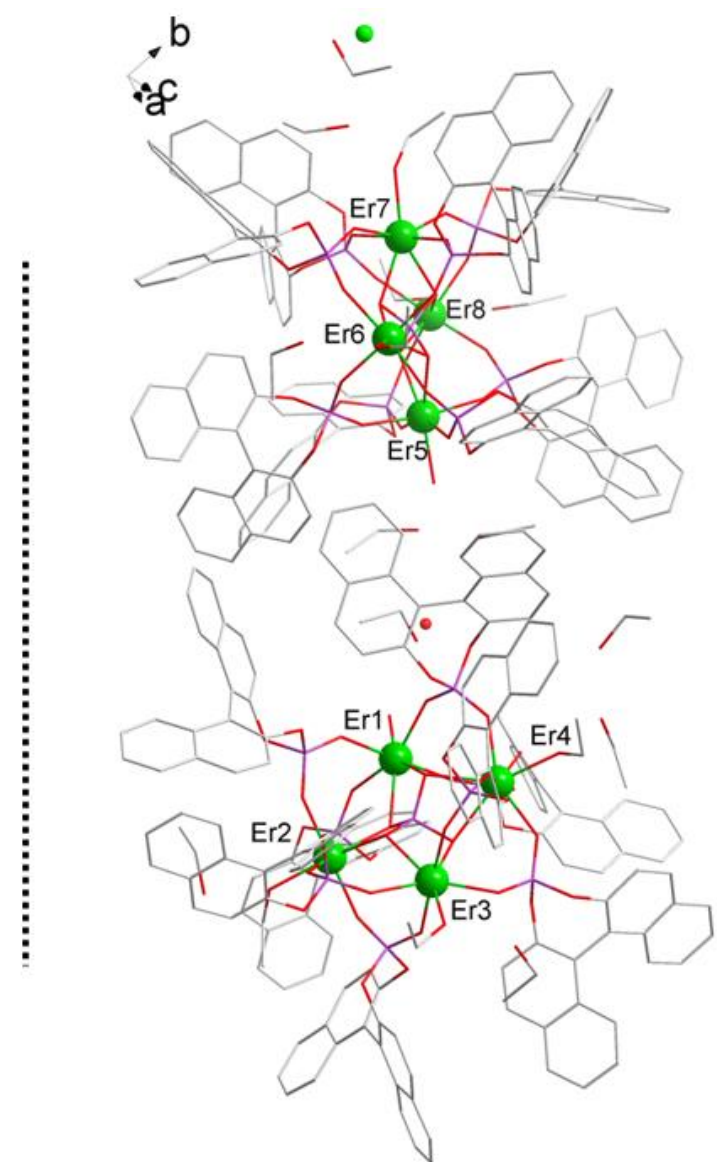

S-1

Figure S4. asymmetric unit of $\boldsymbol{R}-\mathbf{2}$. and $\boldsymbol{S}$-2. Color code: $\mathrm{Er}$, green; O, red; $\mathrm{P}$, violet; $\mathrm{Cl}$, bright green; C, gray. 


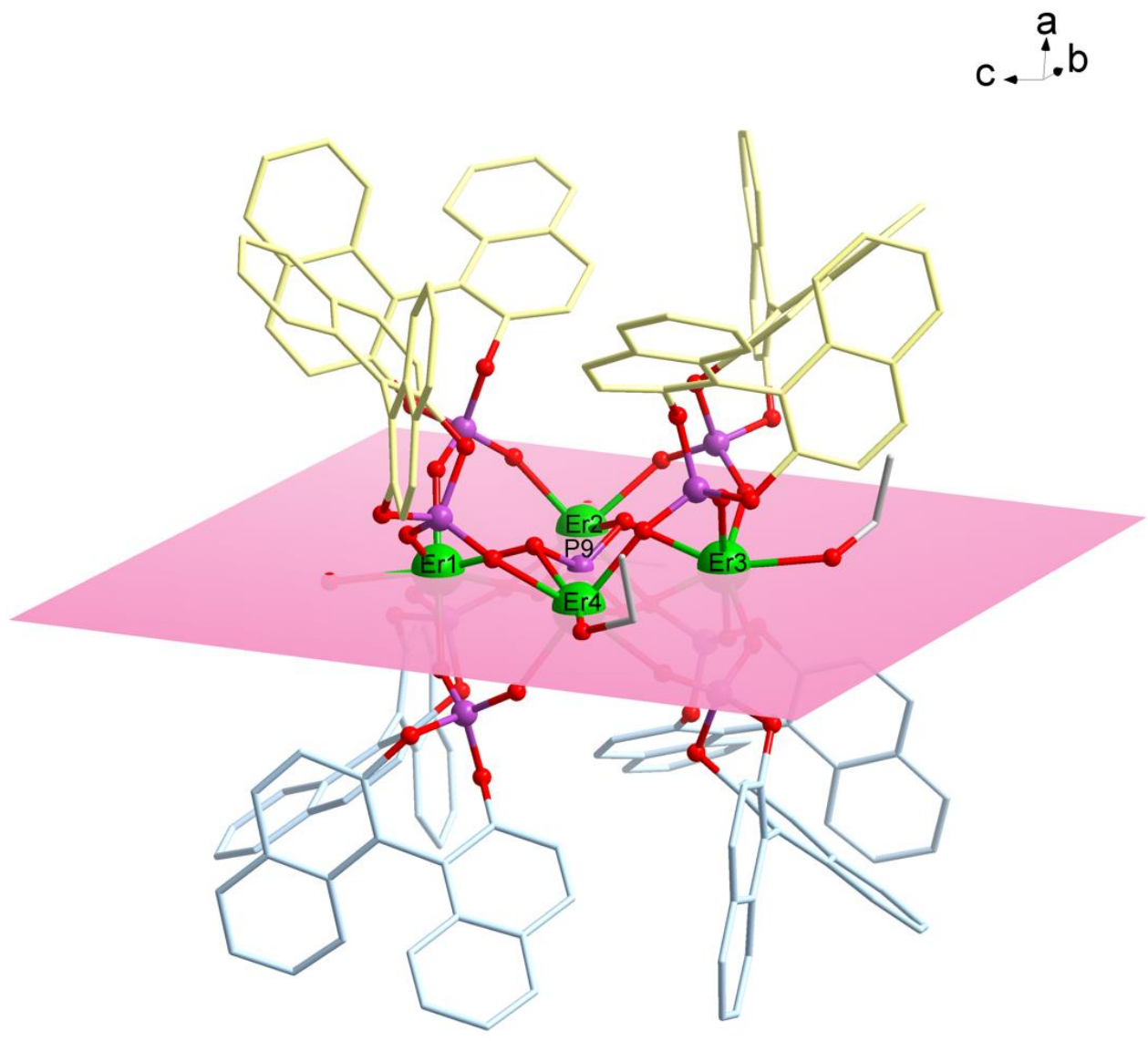

Figure S5. Side view of $\boldsymbol{R}-\mathbf{2}$, illustrating Er1, Er2, Er3, Er4, and P9 are on one plane. Color code: $\mathrm{Er}$, green; $\mathrm{O}$, red; $\mathrm{P}$, violet; $\mathrm{Cl}$, bright green; $\mathrm{C}$, light yellow for the four ligands on top, light blue for the four ligands at the bottom, gray for ethanol.
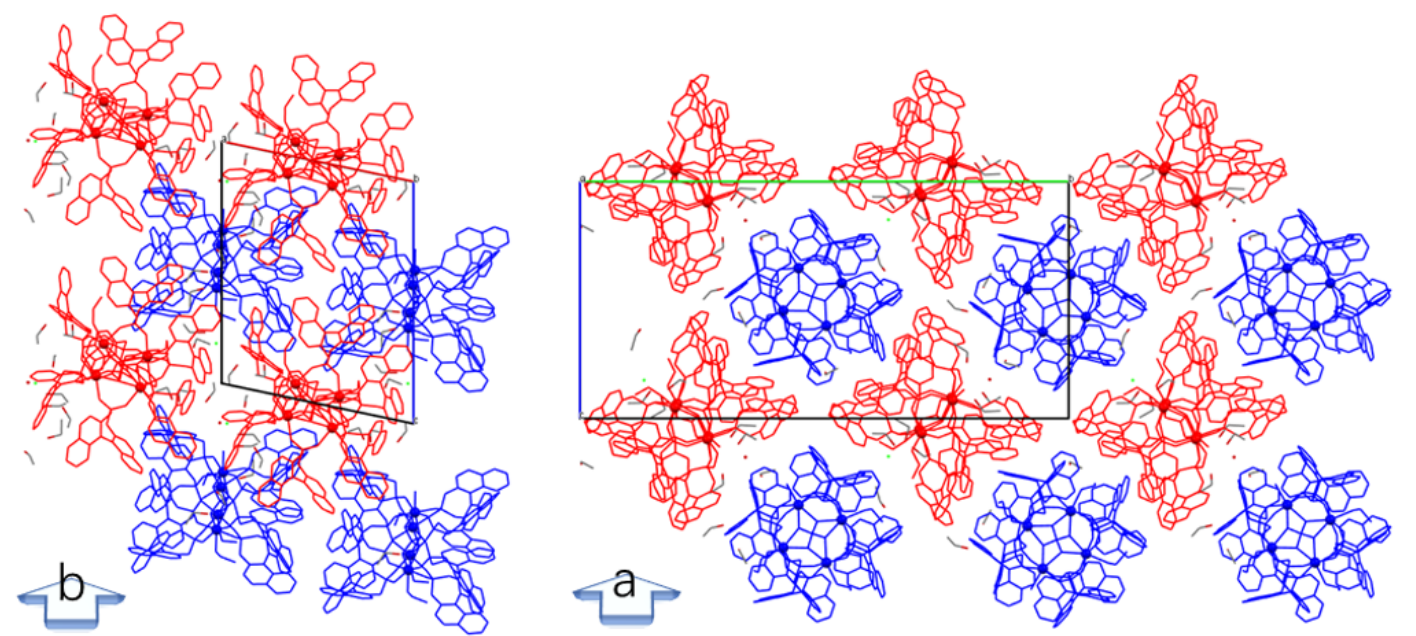

Figure S6. Crystal packing along $\mathrm{b}$ and a axes of $\boldsymbol{R}-\mathbf{2}$. 
Table S1. X-ray Crystallographic Data for $R-1, S-1, R-2$, and $S-2$.

\begin{tabular}{|c|c|c|c|c|}
\hline Compound & $R-1$ & $S-1$ & $R-2$ & $S-2$ \\
\hline Empirical formula & \multirow{2}{*}{\multicolumn{2}{|c|}{$\begin{array}{c}\mathrm{C}_{105} \mathrm{H}_{123} \mathrm{Cl}_{2} \mathrm{Er}_{2} \mathrm{O}_{28.5} \mathrm{P}_{4} \\
2370.33\end{array}$}} & \multirow{2}{*}{\multicolumn{2}{|c|}{$\begin{array}{c}\mathrm{C}_{362} \mathrm{H}_{345} \mathrm{ClEr}_{8} \mathrm{O}_{107} \mathrm{P}_{18} \\
8338.35\end{array}$}} \\
\hline Formula weight & & & & \\
\hline Temperature/K & 120.0 & $100.00(10)$ & $100.00(10)$ & $100.00(10)$ \\
\hline Crystal system & orthorhombic & orthorhombic & monoclinic & monoclinic \\
\hline Space group & $P 2_{1} 2_{1} 2_{1}$ & $P 2_{1} 2_{1} 2_{1}$ & $P 2_{1}$ & $P 2_{1}$ \\
\hline $\mathrm{a} / \AA$ & $14.698(2)$ & $14.69270(10)$ & $18.48733(10)$ & $18.46641(8)$ \\
\hline $\mathrm{b} / \AA \AA$ & $20.890(3)$ & $20.8842(2)$ & $46.11821(19)$ & $46.11573(17)$ \\
\hline $\mathrm{c} / \AA ̊$ & $34.425(5)$ & $34.5457(3)$ & $22.85642(11)$ & $22.85806(11)$ \\
\hline$\beta /{ }^{\circ}$ & 90 & 90 & $101.7501(5)$ & $101.8261(4)$ \\
\hline Volume $/ \AA^{3}$ & $10570(3)$ & $10600.19(15)$ & 19079.08(16) & $19052.57(14)$ \\
\hline $\mathrm{Z}$ & 4 & 4 & 2 & 2 \\
\hline$\rho_{\text {calc }} / \mathrm{g} \mathrm{cm}^{-3}$ & 1.489 & 1.485 & 1.451 & 1.453 \\
\hline$\mu / \mathrm{mm}^{-1}$ & 1.764 & 4.513 & 4.56 & 1.916 \\
\hline $\mathrm{F}(000)$ & 4842.0 & 4844.0 & 8408.0 & 8408.0 \\
\hline Radiation & $\begin{array}{l}\mathrm{Mo} K \alpha \\
(\lambda=071073)\end{array}$ & $\begin{array}{l}\mathrm{CuK \alpha} \\
(\lambda=154184)\end{array}$ & $\begin{array}{l}\mathrm{CuK \alpha} \\
(\lambda=154184)\end{array}$ & $\begin{array}{l}\mathrm{CuK \alpha} \\
(\lambda=154184)\end{array}$ \\
\hline Reflections collected & $\begin{array}{l}(\lambda=0 . / 10 / 3) \\
72136\end{array}$ & $\begin{array}{l}(\lambda=1.54184) \\
70205\end{array}$ & $\begin{array}{l}(\lambda=1.54184) \\
223686\end{array}$ & $\begin{array}{l}(\lambda=1.54184) \\
483036\end{array}$ \\
\hline Independent reflections & 24370 & 17610 & 57543 & 64797 \\
\hline Data/restraints/parameters & $24370 / 58 / 1319$ & $17610 / 73 / 1304$ & $57543 / 357 / 4214$ & $85638 / 85 / 4221$ \\
\hline Goodness-of-fit on $F^{2}$ & 1.061 & 1.127 & 1.021 & 1.030 \\
\hline Final $R$ indexes $[I \geq 2 \sigma(I)]$ & $\begin{array}{l}R_{1}=0.0471 \\
w R_{2}=0.1164\end{array}$ & $\begin{array}{l}R_{1}=0.0614 \\
w R_{2}=0.1634\end{array}$ & $\begin{array}{l}R_{1}=0.0758 \\
w R_{2}=0.2101\end{array}$ & $\begin{array}{l}R_{1}=0.0566 \\
w R_{2}=0.1487\end{array}$ \\
\hline Final $R$ indexes [all data] & $\begin{array}{l}R_{1}=0.0547 \\
w R_{2}=0.1203\end{array}$ & $\begin{array}{l}R_{1}=0.0623 \\
w R_{2}=0.1640\end{array}$ & $\begin{array}{l}R_{1}=0.0790 \\
w R_{2}=0.2123\end{array}$ & $\begin{array}{l}R_{1}=0.0580 \\
w R_{2}=0.1497\end{array}$ \\
\hline Largest diff. peak/hole / e $\AA^{-3}$ & $4.01 /-1.60$ & $3.59 /-1.29$ & $1.60 /-1.17$ & $1.12 /-0.78$ \\
\hline Flack parameter & $0.026(3)$ & $0.024(3)$ & $0.009(9)$ & $0.010(5)$ \\
\hline CCDC number & 1900543 & 1900544 & 1900546 & 1900545 \\
\hline
\end{tabular}


Table S2. Continuous Shape Measures $(\mathrm{CShM})^{1}$ and Continuous Chirality Measures $(\mathrm{CCM})^{2}$ calculations for $\boldsymbol{R}-\mathbf{1}$

\begin{tabular}{|c|c|c|c|c|c|c|c|c|c|c|c|}
\hline \multirow{2}{*}{$\begin{array}{l}\text { Metal } \\
\text { center }\end{array}$} & \multicolumn{7}{|c|}{$\mathrm{CShM}^{\mathrm{a}}$} & \multicolumn{2}{|c|}{$\mathrm{CCM}$} & \multicolumn{2}{|c|}{$\begin{array}{c}\text { Average } \\
\text { CCM }\end{array}$} \\
\hline & HP-7 & HPY-7 & $\begin{array}{c}\text { PBPY- } \\
7\end{array}$ & $\begin{array}{c}\text { COC- } \\
7\end{array}$ & $\begin{array}{c}\text { CTPR- } \\
7\end{array}$ & $\begin{array}{c}\text { JPBPY- } \\
7\end{array}$ & $\begin{array}{c}\text { JETPY- } \\
7\end{array}$ & $1^{\text {st b }}$ & $2^{\text {nd } c}$ & $1^{\mathrm{st} \mathrm{b}}$ & $2^{\text {nd } c}$ \\
\hline Er1 & 34.234 & 20.976 & 4.054 & 1.593 & 0.782 & 7.461 & 20.496 & 0.366 & 1.12 & \multirow{2}{*}{0.243} & \multirow{2}{*}{1.03} \\
\hline Er2 & 36.013 & 19.879 & 4.617 & 1.137 & & & & 0.20 & 944 & & \\
\hline
\end{tabular}

a: HP-7 = Heptagon $\left(D_{7 \mathrm{~h}}\right)$; HPY-7 = Hexagonal pyramid $\left(C_{6 \mathrm{v}}\right)$; PBPY-7 = Pentagonal bipyramid $\left(D_{5 \mathrm{~h}}\right)$; COC-7 $=$ Capped octahedron $\left(C_{3 \mathrm{v}}\right)$; CTPR-7 = Capped trigonal prism $\left(C_{2 \mathrm{v}}\right)$; JPBPY-7 = Johnson pentagonal bipyramid J13 $\left(D_{5 \mathrm{~h}}\right)$; JETPY-7 = Johnson elongated triangular pyramid J7 $\left(C_{3 \mathrm{v}}\right)$. b: First coordination sphere $\mathrm{ErO}_{7}$ c: Second coordination sphere $\mathrm{ErO}_{7} \mathrm{P}_{4} \mathrm{C}_{3}$

Table S3. Continuous Shape Measures $(\mathrm{CShM})^{1}$ and Continuous Chirality Measures $(\mathrm{CCM})^{2}$ calculations for $\boldsymbol{R}-\mathbf{2}$

\begin{tabular}{|c|c|c|c|c|c|c|c|c|c|c|c|c|c|}
\hline \multirow{2}{*}{$\begin{array}{l}\text { Metal } \\
\text { center }\end{array}$} & \multicolumn{7}{|c|}{$\mathrm{CShM}^{*}$} & $\mathrm{CCM}$ & \multicolumn{2}{|c|}{ Average CCM } & $\mathrm{CCM}$ & \multicolumn{2}{|c|}{ Average } \\
\hline & HP-7 & HPY-7 & $\begin{array}{l}\text { PBPY- } \\
7\end{array}$ & $\begin{array}{l}\text { COC- } \\
7\end{array}$ & $\begin{array}{l}\text { CTPR- } \\
7\end{array}$ & $\begin{array}{l}\text { JPBPY- } \\
7\end{array}$ & $\begin{array}{l}\text { JETPY- } \\
7\end{array}$ & \multicolumn{3}{|c|}{$1^{\text {st b }}$} & $2^{\text {nd } c}$ & \multicolumn{2}{|l|}{$2^{\text {nd c }}$} \\
\hline Er1 & 33.674 & 24.454 & 0.555 & 6.419 & 4.965 & 3.527 & 22.198 & 0.0935 & \multirow{4}{*}{0.0656} & \multirow{8}{*}{0.0776} & 0.793 & 0.864 & \multirow{8}{*}{0.895} \\
\hline Er2 & 32.818 & 23.602 & 0.486 & 7.781 & 5.933 & 3.095 & 23.276 & 0.0204 & & & 1.07 & & \\
\hline Er3 & 33.656 & 24.211 & 0.557 & 6.152 & 4.859 & 3.654 & 22.277 & 0.109 & & & 0.858 & & \\
\hline Er4 & 32.408 & 22.546 & 0.794 & 6.715 & 5.456 & 3.389 & 21.418 & 0.0397 & & & 0.732 & & \\
\hline Er5 & 32.600 & 24.360 & 0.368 & 7.979 & 5.939 & 3.058 & 23.170 & 0.0632 & \multirow{4}{*}{0.0897} & & 0.615 & \multirow[t]{4}{*}{0.926} & \\
\hline Er6 & 32.081 & 22.586 & 0.650 & 6.767 & 5.368 & 3.495 & 23.444 & 0.0997 & & & 1.14 & & \\
\hline Er7 & 32.333 & 23.163 & 0.677 & 6.26 & 4.479 & 3.194 & 21.485 & 0.174 & & & 0.919 & & \\
\hline Er8 & 33.059 & 23.285 & 0.741 & 7.247 & 5.554 & 3.759 & 21.315 & 0.0215 & & & 1.031 & & \\
\hline
\end{tabular}

a, b: as Table S2

c: Second coordination sphere $\mathrm{ErO}_{7} \mathrm{P}_{5}$ for $\mathrm{Er} 1$ and $\mathrm{Er} 5, \mathrm{ErO}_{7} \mathrm{P}_{5} \mathrm{C}_{1}$ for the rest. 
Table S4. Continuous Chirality Measures $(\mathrm{CCM})^{2}$ calculations for the ligands $R-\mathrm{L}^{-}$of $\boldsymbol{R}-\mathbf{1}$ and $\boldsymbol{R}-\mathbf{2}$

\begin{tabular}{|c|c|c|c|c|}
\hline Complex & $R-\mathrm{L}^{-*}$ & $\mathrm{CCM}$ & \multicolumn{2}{|c|}{ Average CCM } \\
\hline \multirow{4}{*}{$R-1$} & $\mathrm{P} 1$ & 6.18 & \multirow{4}{*}{\multicolumn{2}{|c|}{6.29}} \\
\hline & P2 & 6.72 & & \\
\hline & P3 & 5.93 & & \\
\hline & $\mathrm{P} 4$ & 6.34 & & \\
\hline \multirow{16}{*}{$R-2$} & $\mathrm{P} 1$ & 6.50 & \multirow{8}{*}{6.03} & \multirow{16}{*}{6.08} \\
\hline & $\mathrm{P} 2$ & 6.72 & & \\
\hline & $\mathrm{P} 3$ & 5.93 & & \\
\hline & $\mathrm{P} 4$ & 6.34 & & \\
\hline & $\mathrm{P} 5$ & 5.53 & & \\
\hline & P6 & 5.79 & & \\
\hline & $\mathrm{P} 7$ & 5.55 & & \\
\hline & P8 & 5.89 & & \\
\hline & $\mathrm{P} 10$ & 6.42 & \multirow{8}{*}{6.12} & \\
\hline & P11 & 6.33 & & \\
\hline & $\mathrm{P} 12$ & 5.81 & & \\
\hline & $\mathrm{P} 13$ & 6.03 & & \\
\hline & P14 & 6.69 & & \\
\hline & P15 & 6.11 & & \\
\hline & P16 & 6.10 & & \\
\hline & $\mathrm{P} 17$ & 5.45 & & \\
\hline
\end{tabular}

* $R$ - $\mathrm{L}^{-}$ligands $\left(\mathrm{C}_{20} \mathrm{H}_{12} \mathrm{O}_{4} \mathrm{P}\right)$ with $\mathrm{P} 1-8$ and $\mathrm{P} 10-17$. $\mathrm{P} 9$ and $\mathrm{P} 18$ belong to the two template $\mathrm{PO}_{4}{ }^{-}$ anions.

Table S5. Selected bonds lengths $[\AA]$ for $\boldsymbol{R}-\mathbf{1}$

\begin{tabular}{|c|c|c|c|}
\hline Type & Atoms & Bond length & Average \\
\hline \multirow{4}{*}{ Er1-O(L) } & Er1-O1 & $2.283(5)$ & \multirow{4}{*}{$2.264(6)$} \\
\hline & Er1-O5 & $2.266(6)$ & \\
\hline & Er1-O9 & $2.280(6)$ & \\
\hline & Er1-O13 & $2.228(5)$ & \\
\hline \multirow{3}{*}{ Er1-O(EtOH) } & Er1-O17 & $2.356(7)$ & \multirow{3}{*}{$2.347(7)$} \\
\hline & Er1-O18 & $2.317(8)$ & \\
\hline & Er1-O19 & $2.369(6)$ & \\
\hline \multirow{4}{*}{ Er2-O(L) } & Er2-O2 & $2.250(7)$ & \multirow{4}{*}{$2.258(6)$} \\
\hline & Er2-O6 & $2.263(6)$ & \\
\hline & Er2-O10 & $2.242(5)$ & \\
\hline & Er2-O14 & $2.277(6)$ & \\
\hline \multirow{3}{*}{$\mathrm{Er} 2-\mathrm{O}(\mathrm{EtOH})$} & Er2-O20 & $2.287(7)$ & \multirow{3}{*}{$2.295(7)$} \\
\hline & Er2-O21 & $2.307(7)$ & \\
\hline & Er2-O22 & $2.290(7)$ & \\
\hline
\end{tabular}


Table S6. Selected bonds angles $\left[^{\circ}\right]$ for $\boldsymbol{R}-\mathbf{1}$

\begin{tabular}{|c|c|c|c|}
\hline Type & Atoms & Bond Angle & Average \\
\hline \multirow{4}{*}{$\mathrm{O}\left(\mathrm{L}_{\text {neighbor }}\right)-\mathrm{Er} 1-\mathrm{O}\left(\mathrm{L}_{\text {neighbor }}\right)$} & O5-Er1-O1 & $76.8(2)$ & \multirow{4}{*}{$78.9(2)$} \\
\hline & O5-Er1-O9 & $75.9(2)$ & \\
\hline & O13-Er1-O1 & $79.5(2)$ & \\
\hline & O13-Er1-O9 & $83.5(2)$ & \\
\hline \multirow{2}{*}{$\mathrm{O}\left(\mathrm{L}_{\text {diagonal }}\right)-\mathrm{Er} 1-\mathrm{O}\left(\mathrm{L}_{\text {diagonal }}\right)$} & O1-Er1-O9 & $136.4(2)$ & \multirow{2}{*}{$126.8(2)$} \\
\hline & O5-Er1-O13 & $117.1(2)$ & \\
\hline \multirow{3}{*}{$\mathrm{O}(\mathrm{EtOH})-\mathrm{Er} 1-\mathrm{O}(\mathrm{EtOH})$} & O17-Er1-O18 & $78.2(3)$ & \multirow{3}{*}{$76.8(2)$} \\
\hline & O18-Er1-O19 & $79.3(2)$ & \\
\hline & O19-Er1-O17 & $72.8(2)$ & \\
\hline \multirow{4}{*}{$\mathrm{O}\left(\mathrm{L}_{\text {neighbor }}\right)-\mathrm{Er} 2-\mathrm{O}\left(\mathrm{L}_{\text {neighbor }}\right)$} & O2-Er2-O6 & $76.2(2)$ & \multirow{4}{*}{$77.2(2)$} \\
\hline & O2-Er2-O14 & $76.9(2)$ & \\
\hline & O10-Er2-O6 & $79.6(2)$ & \\
\hline & O10-Er2-O14 & $75.9(2)$ & \\
\hline \multirow{2}{*}{$\mathrm{O}\left(\mathrm{L}_{\text {diagonal }}\right)-\mathrm{Er} 2-\mathrm{O}\left(\mathrm{L}_{\text {diagonal }}\right)$} & O10-Er2-O2 & $110.9(2)$ & \multirow{2}{*}{$122.4(2)$} \\
\hline & O6-Er2-O14 & $133.9(2)$ & \\
\hline \multirow{3}{*}{$\mathrm{O}(\mathrm{EtOH})-\mathrm{Er} 2-\mathrm{O}(\mathrm{EtOH})$} & O20-Er2-O21 & $83.8(3)$ & \multirow{3}{*}{$78.7(3)$} \\
\hline & O20-Er2-O22 & $77.5(3)$ & \\
\hline & $\mathrm{O} 22-\mathrm{Er} 2-\mathrm{O} 21$ & $74.8(3)$ & \\
\hline
\end{tabular}

Table S7. Zenith angles (angles of ligand-metal-calculated main magnetic axes) [ $\left.{ }^{\circ}\right]$ for $\boldsymbol{R}-\mathbf{1}$

\begin{tabular}{|c|c|c|c|}
\hline Type & Atoms & Angle & Average \\
\hline \multirow{4}{*}{ O(L)-Er1-axis* } & O1-Er1-axis & 81.0 & \multirow{4}{*}{64.7} \\
\hline & O5-Er1-axis & 72.3 & \\
\hline & O9-Er1-axis & 58.5 & \\
\hline & O13-Er1-axis & 46.9 & \\
\hline \multirow{3}{*}{$\mathrm{O}(\mathrm{EtOH})$-Er1-axis } & O17-Er1-axis & 40.3 & \multirow{3}{*}{48.8} \\
\hline & O18-Er1-axis & 39.0 & \\
\hline & O19-Er1-axis & 67.2 & \\
\hline \multirow{4}{*}{$\mathrm{O}(\mathrm{L})$-Er2-axis } & O2-Er2-axis & 77.6 & \multirow{4}{*}{63.0} \\
\hline & O6-Er2-axis & 56.3 & \\
\hline & O10-Er2-axis & 36.3 & \\
\hline & O14-Er2-axis & 81.6 & \\
\hline \multirow{3}{*}{$\mathrm{O}(\mathrm{EtOH})$-Er2-axis } & O20-Er2-axis & 36.6 & \multirow{3}{*}{51.0} \\
\hline & O21-Er2-axis & 75.9 & \\
\hline & O22-Er2-axis & 40.59 & \\
\hline
\end{tabular}

* The main magnetic axes of the ground Kramers doublet obtained from the $a b$ initio calculations. 
Table S8. Selected bonds lengths $[\AA]$ for $\boldsymbol{R}-\mathbf{2}$.

\begin{tabular}{|c|l|l|l|l|l|}
\hline \multicolumn{2}{|c|}{ Er-O $(R$-L $)$} & \multicolumn{2}{c|}{ Er-O $\left(\mathrm{PO}_{4}{ }^{3-}\right)$} & \multicolumn{2}{c|}{ Er-O $\left(\mathrm{EtOH} / \mathrm{H}_{2} \mathrm{O}\right)$} \\
\hline Average & $2.240(14)$ & Average & $2.430(12)$ & Average & $2.304(18)$ \\
\hline Er1-O1 & $2.260(12)$ & Er1-O33 & $2.397(11)$ & Er1-O37 & $2.288(13)$ \\
\hline Er1-O5 & $2.227(12)$ & Er1-O34 & $2.445(12)$ & Er2-O38 & $2.315(18)$ \\
\hline Er1-O26 & $2.241(12)$ & Er2-O34 & $2.427(12)$ & Er3-O39 & $2.297(12)$ \\
\hline Er1-O30 & $2.263(11)$ & Er2-O35 & $2.452(12)$ & Er4-O40 & $2.290(14)$ \\
\hline Er2-O2 & $2.247(12)$ & Er3-O35 & $2.417(12)$ & Er5-O77 & $2.278(14)$ \\
\hline Er2-O6 & $2.198(13)$ & Er3-O36 & $2.388(12)$ & Er6-O78 & $2.333(13)$ \\
\hline Er2-O9 & $2.205(13)$ & Er4-O33 & $2.463(12)$ & Er7-O79 & $2.322(15)$ \\
\hline Er2-O13 & $2.278(14)$ & Er4-O36 & $2.464(12)$ & Er8-O80 & $2.312(14)$ \\
\hline Er3-O10 & $2.251(12)$ & Er5-O73 & $2.418(12)$ & & \\
\hline Er3-O14 & $2.254(13)$ & Er5-O74 & $2.438(11)$ & & \\
\hline Er3-O17 & $2.253(13)$ & Er6-O74 & $2.421(11)$ & & \\
\hline Er3-O21 & $2.236(12)$ & Er6-O75 & $2.435(11)$ & & \\
\hline Er4-O18 & $2.237(12)$ & Er7-O75 & $2.423(12)$ & & \\
\hline Er4-O22 & $2.195(11)$ & Er7-O76 & $2.452(11)$ & & \\
\hline Er4-O25 & $2.204(12)$ & Er8-O73 & $2.405(12)$ & & \\
\hline Er4-O29 & $2.253(12)$ & Er8-O76 & $2.435(10)$ & & \\
\hline Er5-O41 & $2.293(11)$ & & & & \\
\hline Er5-O45 & $2.214(12)$ & & & & \\
\hline Er5-O66 & $2.230(13)$ & & & & \\
\hline Er5-O70 & $2.309(13)$ & & & & \\
\hline Er6-O42 & $2.227(12)$ & & & & \\
\hline Er6-O46 & $2.224(13)$ & & & & \\
\hline Er6-O49 & $2.241(13)$ & & & & \\
\hline Er6-O53 & $2.277(13)$ & & & & \\
\hline Er7-O50 & $2.172(11)$ & & & & \\
\hline Er7-O54 & $2.276(13)$ & & & & \\
\hline Er7-O57 & $2.273(13)$ & & & & \\
\hline Er7-O61 & $2.213(12)$ & & & & \\
\hline Er8-O58 & $2.241(12)$ & & & & \\
\hline Er8-O62 & $2.226(12)$ & & & & \\
\hline Er8-O65 & $2.246(12)$ & & & & \\
\hline Er8-O69 & $2.230(13)$ & & & & \\
\hline
\end{tabular}


Table S9. Er...Er distances $[\AA]$ for $\boldsymbol{R}-\mathbf{2}$.

\begin{tabular}{|c|c|c|c|c|}
\hline type & Metal centers & distance & Ave & age \\
\hline \multirow{8}{*}{ neighbor Er } & Er1...Er2 & $4.330(1)$ & \multirow{4}{*}{$4.318(1)$} & \multirow{8}{*}{$4.317(1)$} \\
\hline & Er2...Er3 & $4.310(1)$ & & \\
\hline & Er3...Er4 & $4.334(1)$ & & \\
\hline & Er4...Er1 & $4.300(1)$ & & \\
\hline & Er5...Er6 & $4.362(1)$ & \multirow{4}{*}{$4.315(1)$} & \\
\hline & Er6...Er7 & $4.291(1)$ & & \\
\hline & Er7...Er8 & $4.319(1)$ & & \\
\hline & Er8...Er5 & $4.289(1)$ & & \\
\hline \multirow{4}{*}{ diagonal Er } & Er1...Er3 & $6.081(1)$ & \multirow{2}{*}{$6.102(1)$} & \multirow{4}{*}{$6.104(1)$} \\
\hline & Er2...Er4 & $6.133(1)$ & & \\
\hline & Er5...Er7 & $6.095(1)$ & \multirow{2}{*}{$6.107(2)$} & \\
\hline & Er6...Er8 & $6.108(1)$ & & \\
\hline intermolecular & Er1...Er5 & $11.760(1)$ & & \\
\hline
\end{tabular}

(a)

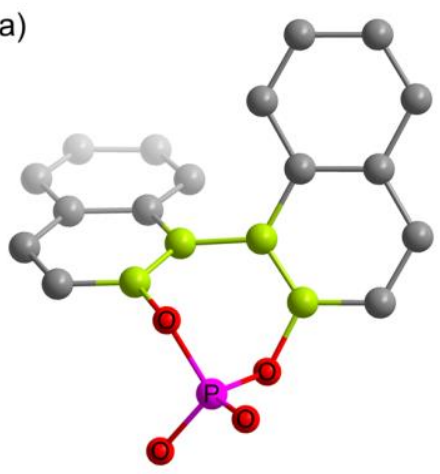

torsion angles: $\Phi 1=53.1(4)^{\circ}$ (b)

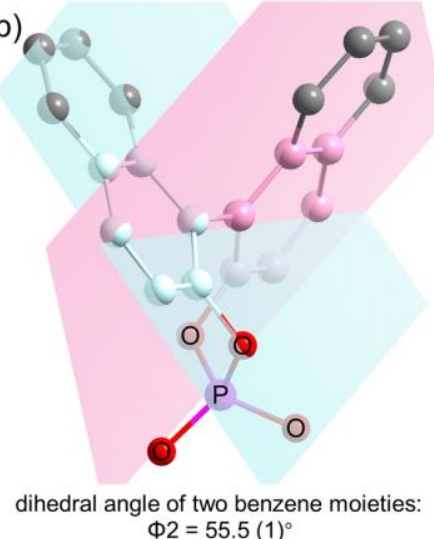

(c)

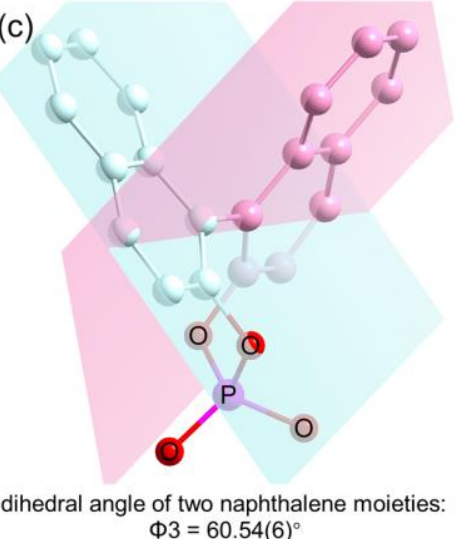
Ф3 $=60.54(6)^{\circ}$

Figure S7. Torsion angle (a, $\Phi 1$ ), dihedral angles of two benzene (b, $\Phi 2)$ and two naphthalene (c, Ф3) moieties of free ligand $S$-HL (CCDC: 182411) ${ }^{3}$, measured using Diamond Version 4.5.3. 


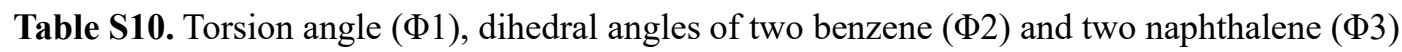
moieties $\left[{ }^{\circ}\right]$ for $\boldsymbol{R}-\mathbf{1}$ and $\boldsymbol{R}-\mathbf{2}$ (see Figure S7), measured using Diamond Version 4.5.3.

\begin{tabular}{|c|c|c|c|c|c|c|c|c|c|c|}
\hline Complex & $R-\mathrm{L}^{-*}$ & $\Phi 1$ & \multicolumn{2}{|c|}{$\begin{array}{c}\text { Average } \\
\Phi 1\end{array}$} & $\Phi 2$ & \multicolumn{2}{|c|}{$\begin{array}{c}\text { Average } \\
\Phi 2\end{array}$} & $\Phi 3$ & \multicolumn{2}{|c|}{$\begin{array}{c}\text { Average } \\
\Phi 3\end{array}$} \\
\hline \multirow{4}{*}{$R-1$} & $\mathrm{P} 1$ & $-50(1)$ & \multirow{4}{*}{\multicolumn{2}{|c|}{$-51(1)$}} & $54.1(3)$ & \multirow{4}{*}{\multicolumn{2}{|c|}{$53.5(3)$}} & $56.3(2)$ & \multirow{4}{*}{\multicolumn{2}{|c|}{$56.9(2)$}} \\
\hline & $\mathrm{P} 2$ & $-51(1)$ & & & $53.9(3)$ & & & $59.0(2)$ & & \\
\hline & P3 & $-50(1)$ & & & $51.9(3)$ & & & $55.0(1)$ & & \\
\hline & $\mathrm{P} 4$ & $-52(1)$ & & & $54.2(3)$ & & & $57.3(2)$ & & \\
\hline \multirow{16}{*}{$R-2$} & $\mathrm{P} 1$ & $-54(3)$ & \multirow{8}{*}{$-54(3)$} & \multirow{16}{*}{$-54(3)$} & $57.2(7)$ & \multirow{8}{*}{$54.6(17)$} & \multirow{16}{*}{$55.8(17)$} & $63.8(4)$ & \multirow{8}{*}{$58.6(7)$} & \multirow{16}{*}{$60.1(7)$} \\
\hline & $\mathrm{P} 2$ & $-56(2)$ & & & $55.9(10)$ & & & $59.3(5)$ & & \\
\hline & P3 & $-51(3)$ & & & $51.1(17)$ & & & $54.5(7)$ & & \\
\hline & $\mathrm{P} 4$ & $-52(3)$ & & & $51.8(7)$ & & & $56.1(4)$ & & \\
\hline & P5 & $-55(2)$ & & & $54.8(8)$ & & & $58.0(4)$ & & \\
\hline & P6 & $-60(3)$ & & & $57.6(7)$ & & & $60.0(4)$ & & \\
\hline & P7 & $-51(2)$ & & & $53.2(9)$ & & & $57.1(4)$ & & \\
\hline & P8 & $-53(3)$ & & & $54.9(8)$ & & & $59.8(4)$ & & \\
\hline & P10 & $-56(2)$ & \multirow{8}{*}{$-53(3)$} & & $58.5(6)$ & \multirow{8}{*}{$57.1(8)$} & & $62.9(3)$ & \multirow{8}{*}{$61.6(4)$} & \\
\hline & P11 & $-53(2)$ & & & $58.3(8)$ & & & $63.2(3)$ & & \\
\hline & $\mathrm{P} 12$ & $-53(2)$ & & & $58.1(7)$ & & & $61.3(4)$ & & \\
\hline & P13 & $-50(3)$ & & & $52.3(8)$ & & & $58.3(4)$ & & \\
\hline & P14 & $-52(2)$ & & & $59.6(6)$ & & & $65.4(3)$ & & \\
\hline & $\mathrm{P} 15$ & $-55(2)$ & & & $58.4(8)$ & & & $62.7(4)$ & & \\
\hline & $\mathrm{P} 16$ & $-53(3)$ & & & $57.6(6)$ & & & $62.1(4)$ & & \\
\hline & P17 & $-52(2)$ & & & $53.7(6)$ & & & $56.7(4)$ & & \\
\hline
\end{tabular}

* $R$ - $\mathrm{L}^{-}$ligands $\left(\mathrm{C}_{20} \mathrm{H}_{12} \mathrm{O}_{4} \mathrm{P}\right)$ with $\mathrm{P} 1-8$ and $\mathrm{P} 10-17 . \mathrm{P} 9$ and $\mathrm{P} 18$ belong to the two template $\mathrm{PO}_{4}^{-}$ anions. 


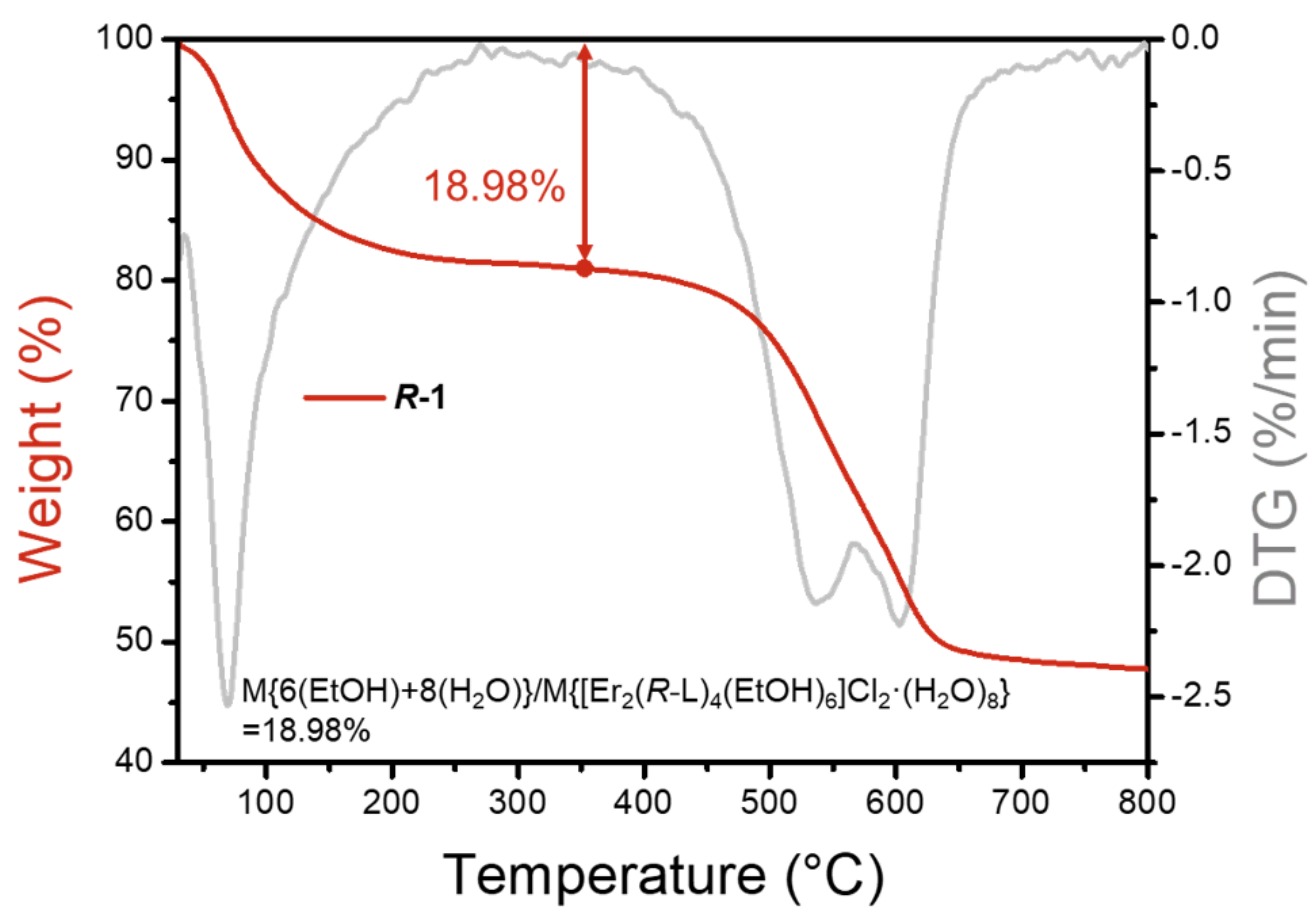

Figure S8. Thermogravimetry (TG) and differential thermal gravity (DTG) curves of $\boldsymbol{R}$-1. The crystalline sample was freshly filtered.

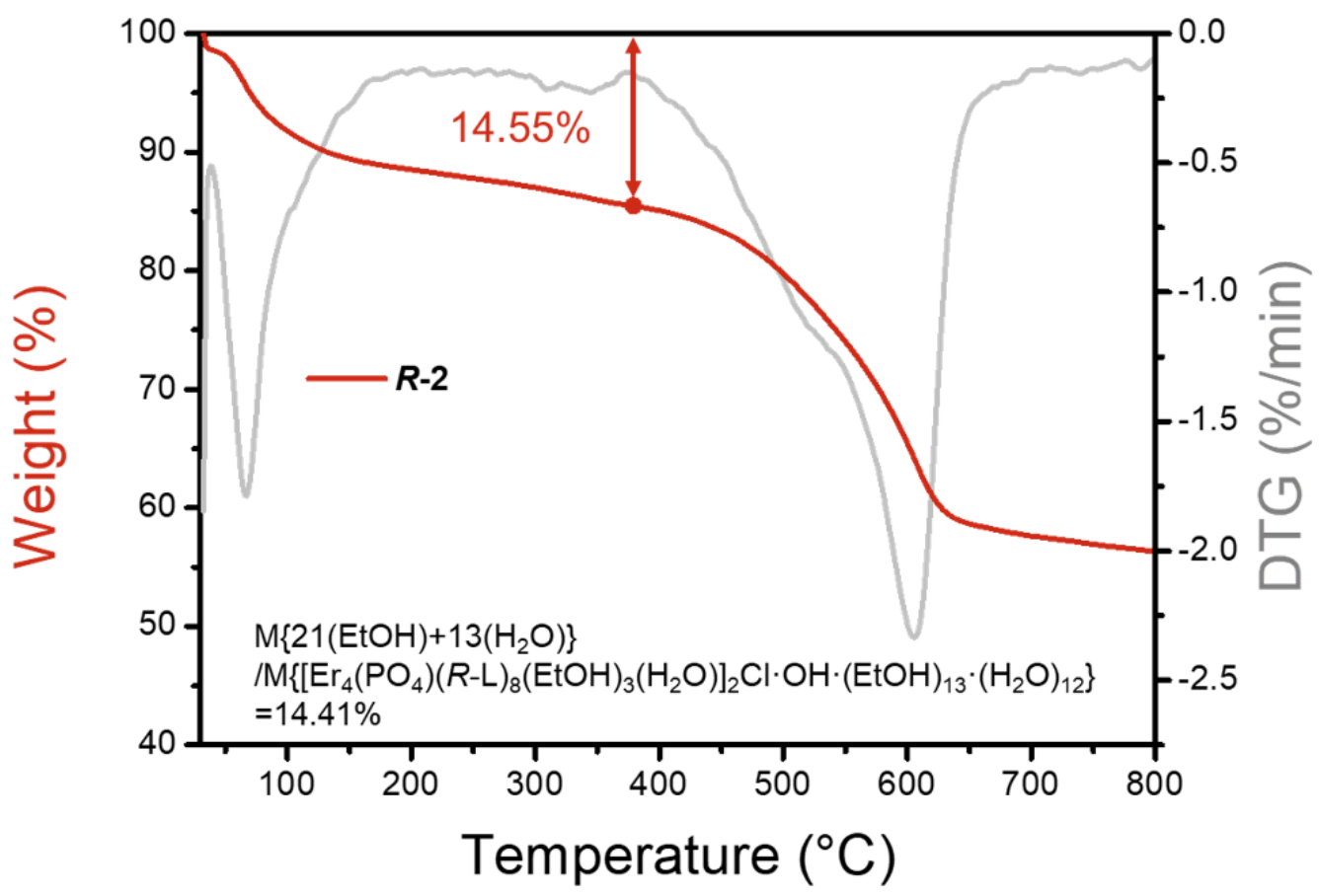

Figure S9. Thermogravimetry (TG) and differential thermal gravity (DTG) curves of $\boldsymbol{R}-\mathbf{2}$. The crystalline sample was freshly filtered. 


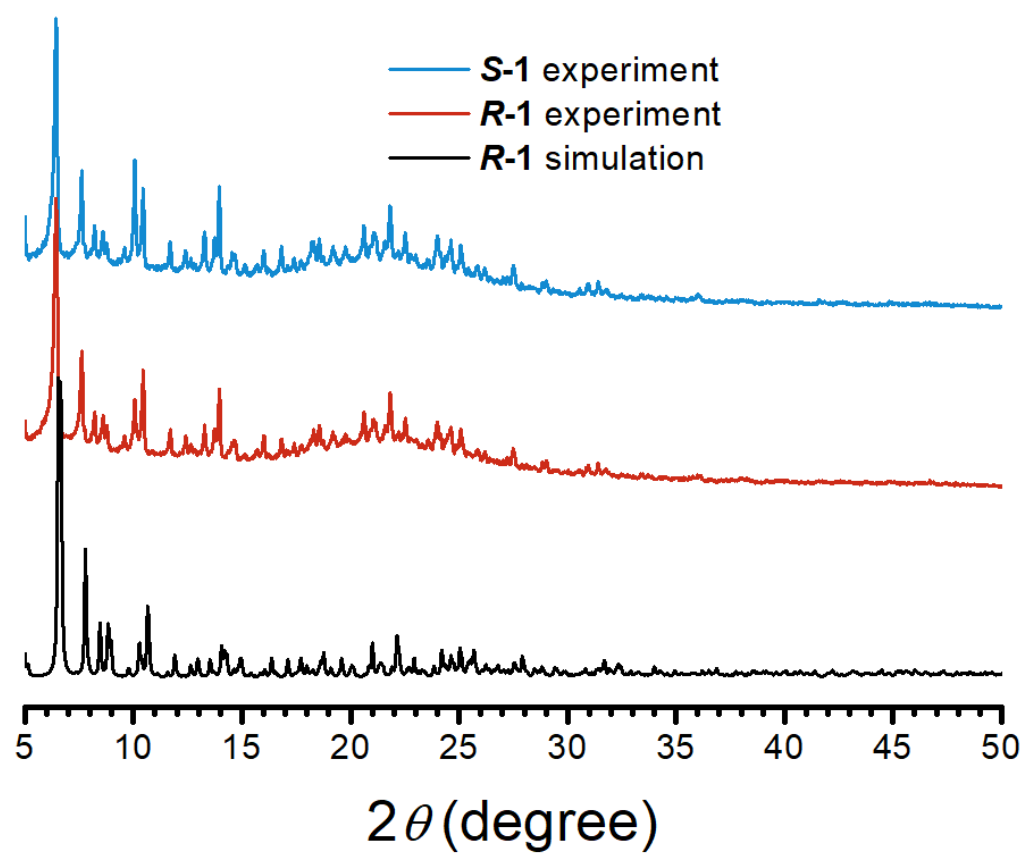

Figure S10. Experimental and simulated X-ray powder diffraction (PXRD) patterns of $\boldsymbol{R}-\mathbf{1}$ and $\boldsymbol{S}$ 1.

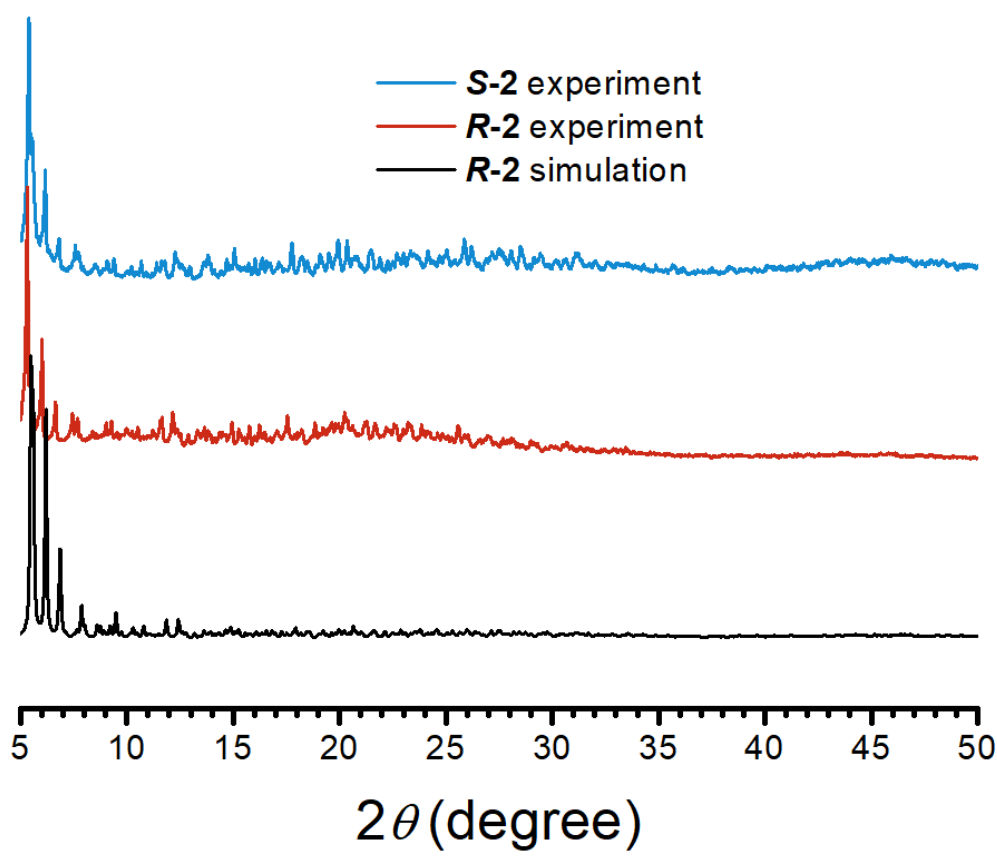

Figure S11. Experimental and simulated X-ray powder diffraction (PXRD) patterns of $\boldsymbol{R}-\mathbf{2}$ and $\boldsymbol{S}$ 2. 


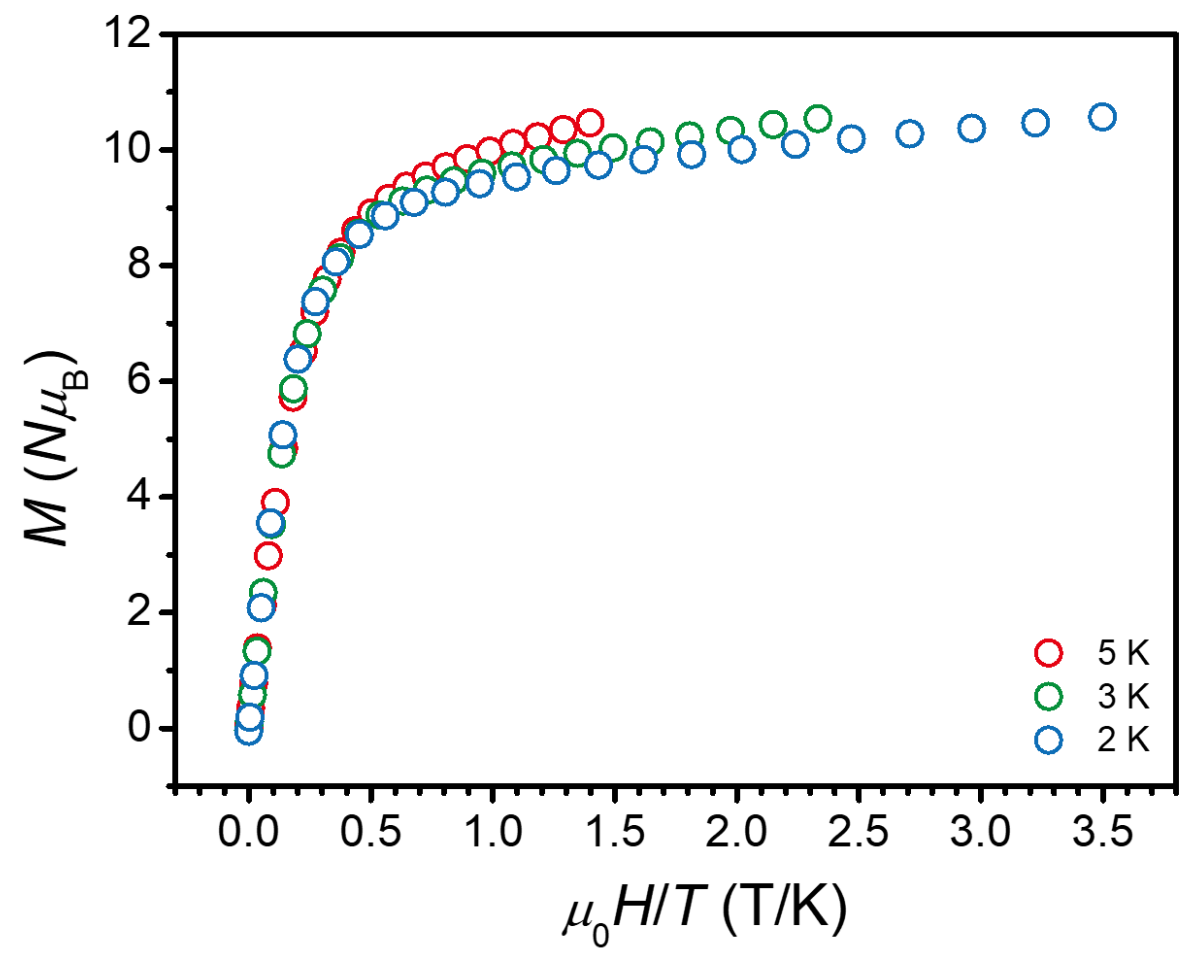

Figure S12. Plots of reduced magnetization $(M / N \beta)$ vs $H / T$ for $\boldsymbol{R}-\mathbf{1}$.

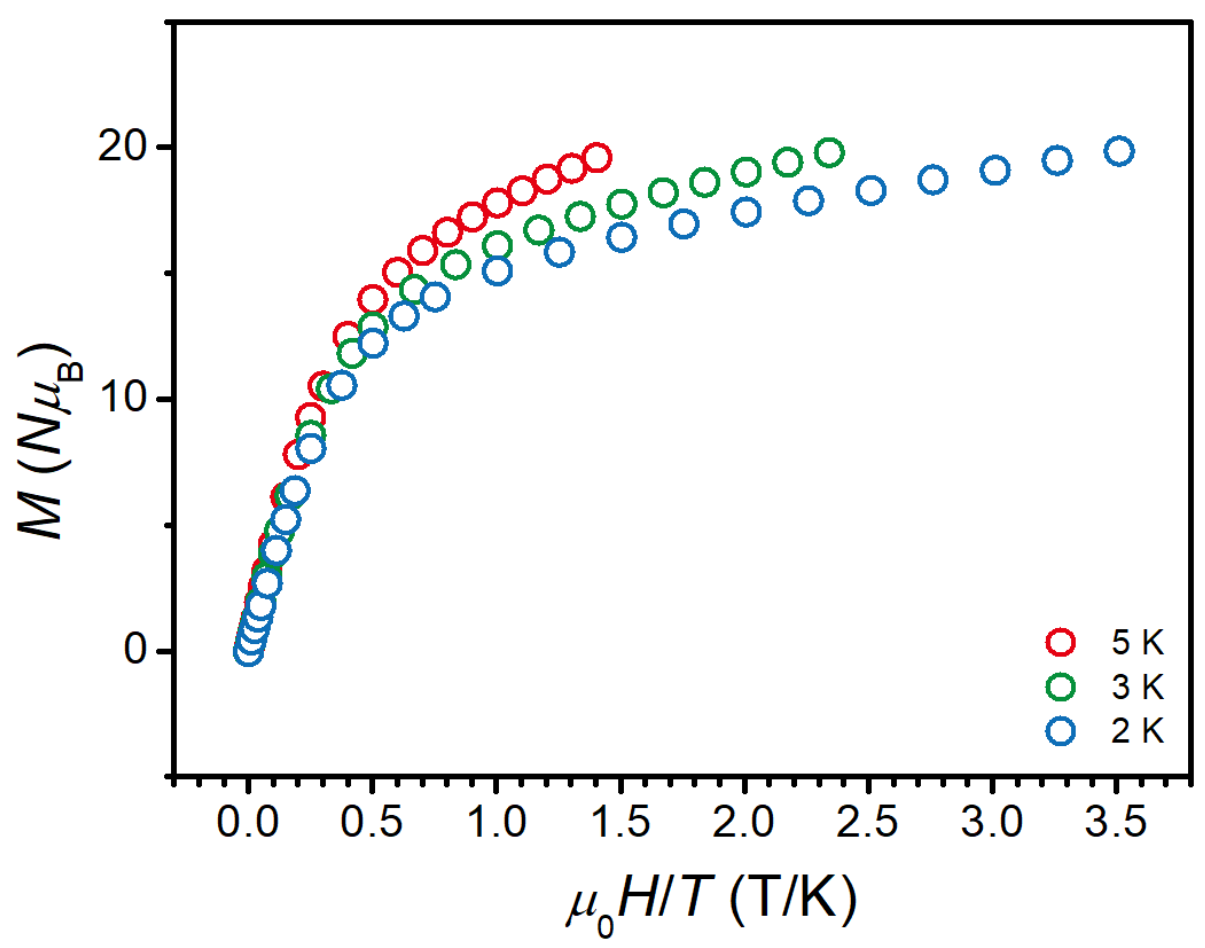

Figure S13. Plots of reduced magnetization $(M / N \beta)$ vs $H / T$ for $\boldsymbol{R}-\mathbf{2}$. 


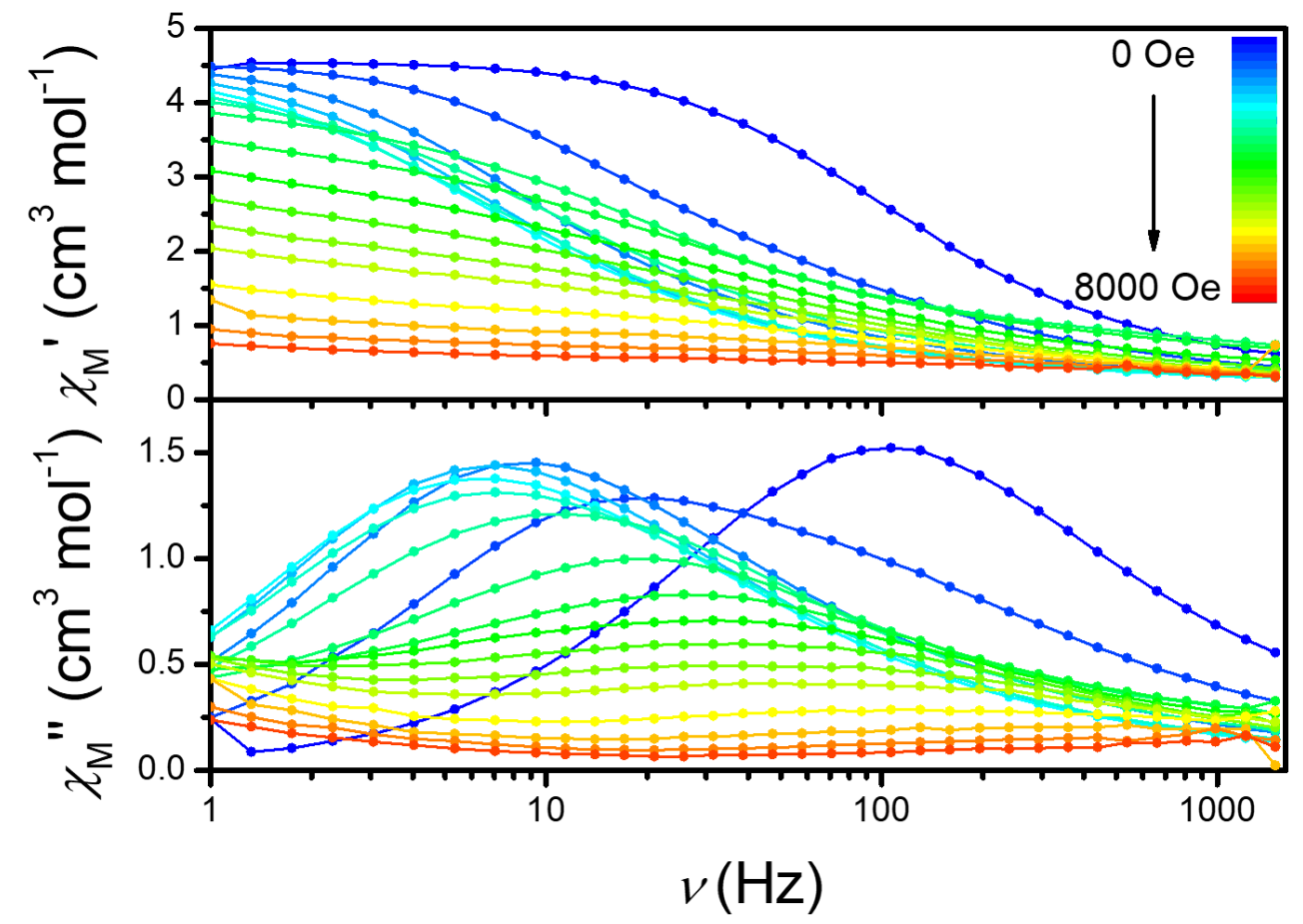

Figure S14. Scan field of the frequency dependence of $\chi_{\mathrm{M}^{\prime}}$ and $\chi_{\mathrm{M}}$ " for $\boldsymbol{R}-\mathbf{1}$ at $3 \mathrm{~K}$ between $0-$ 8000 Oe. The solid lines are guides to the eyes.

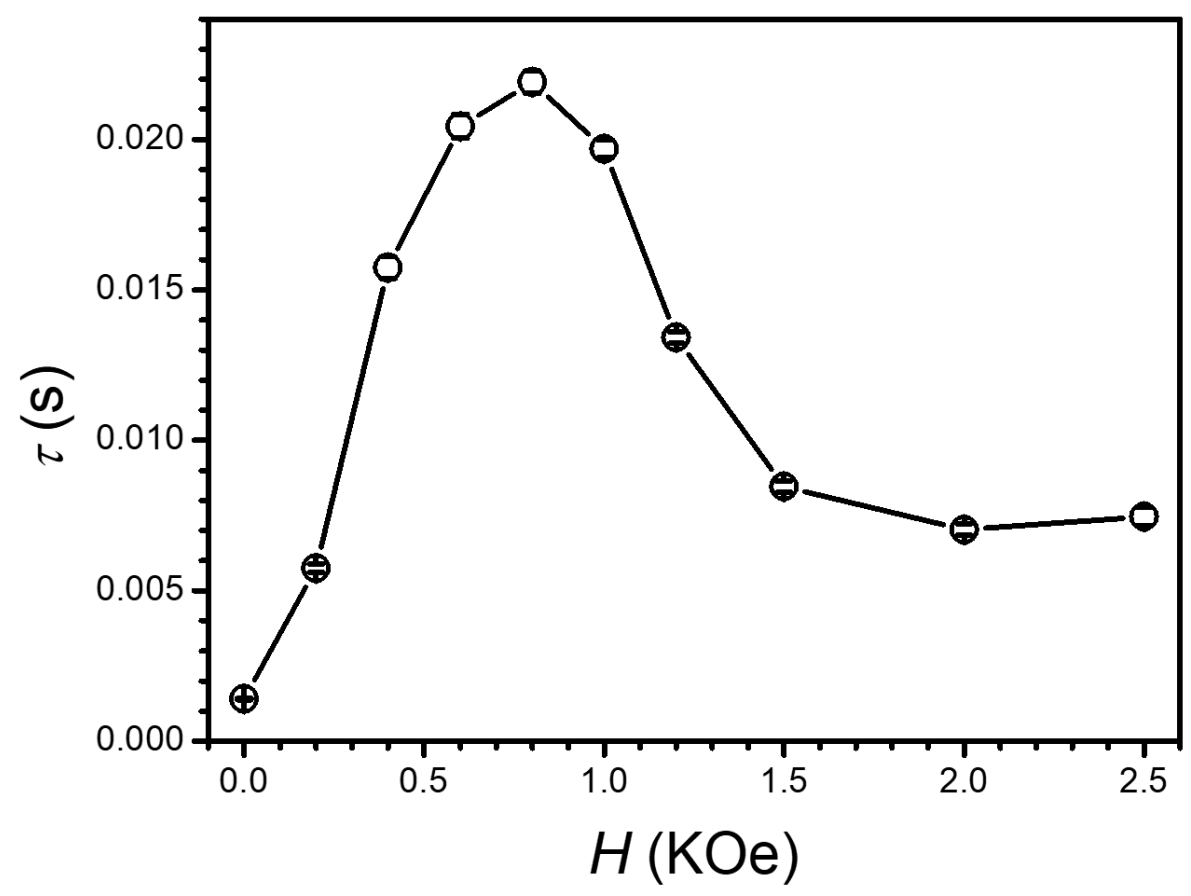

Figure S15. Field dependence of the relaxation time $\tau$ for $\boldsymbol{R}-\mathbf{1}$. 


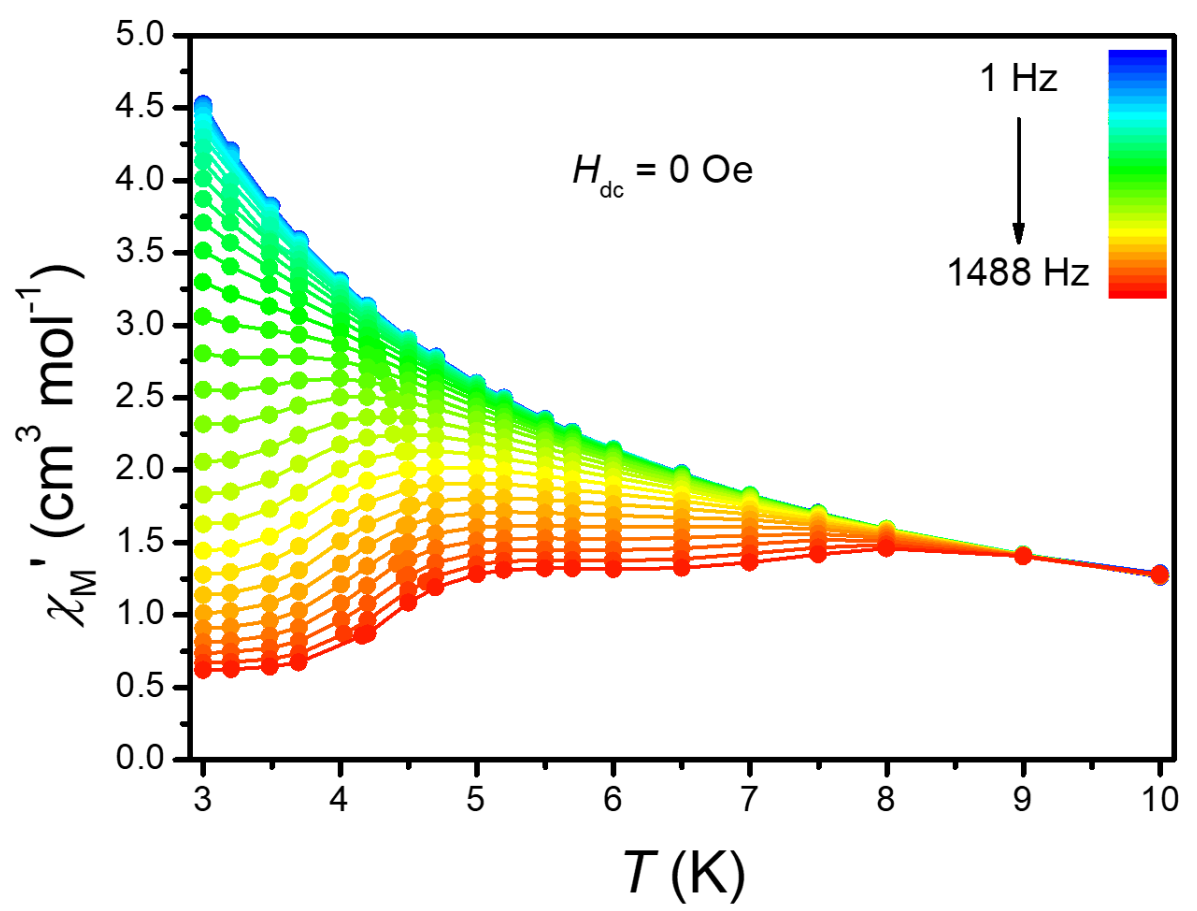

Figure S16. Temperature dependences of $\chi_{\mathrm{M}^{\prime}}$ measured between $1 \mathrm{~Hz}$ and $1488 \mathrm{~Hz}$ for $\boldsymbol{R}-\mathbf{1}$ under a zero field. The solid lines are guides to the eyes.

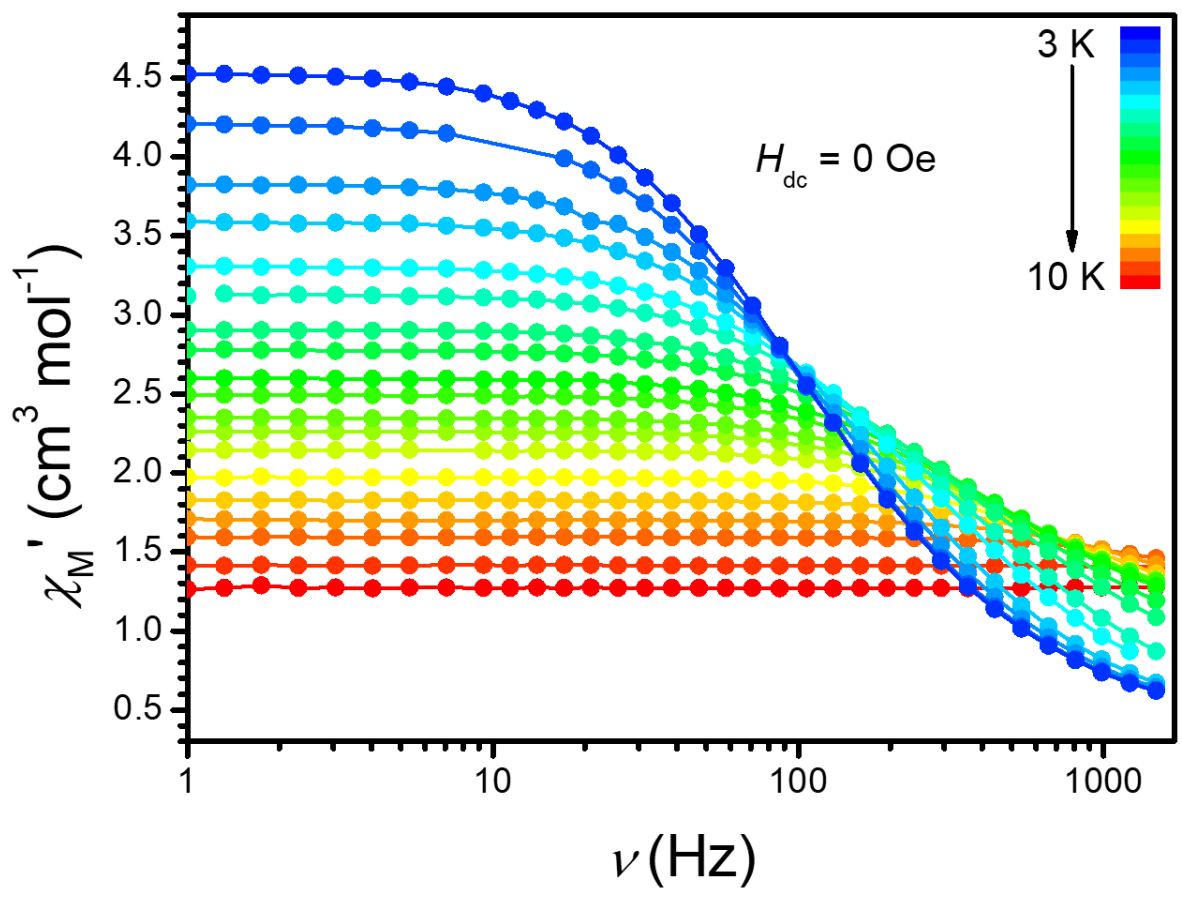

Figure S17. Frequency dependences of $\chi_{\mathrm{M}}$ ' measured between 3 and $10 \mathrm{~K}$ for $\boldsymbol{R}-\mathbf{1}$ under a zero field. The solid lines are guides to the eyes. 


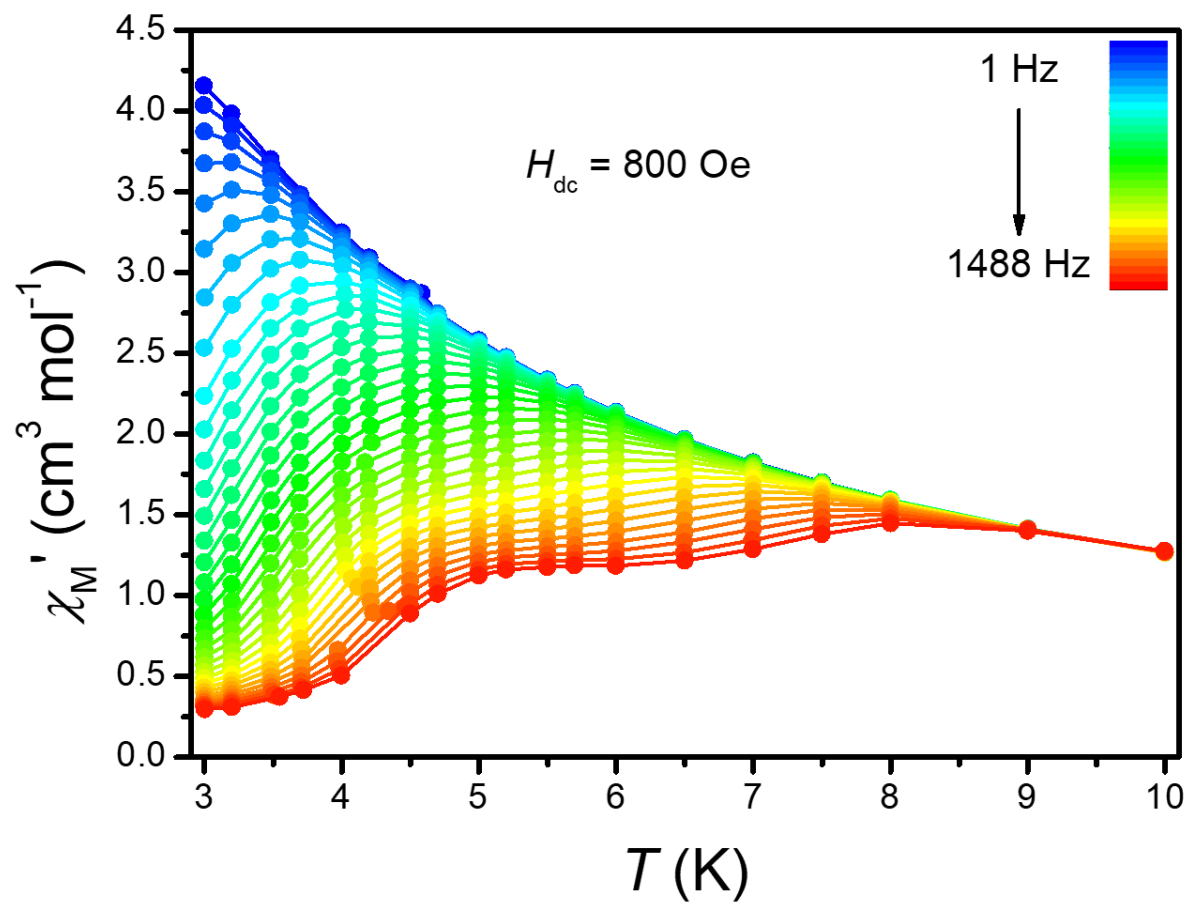

Figure S18. Temperature dependences of $\chi_{\mathrm{M}}$ ' measured between $1 \mathrm{~Hz}$ and $1488 \mathrm{~Hz}$ for $\boldsymbol{R}-\mathbf{1}$ under an 800 Oe field. The solid lines are guides to the eyes.

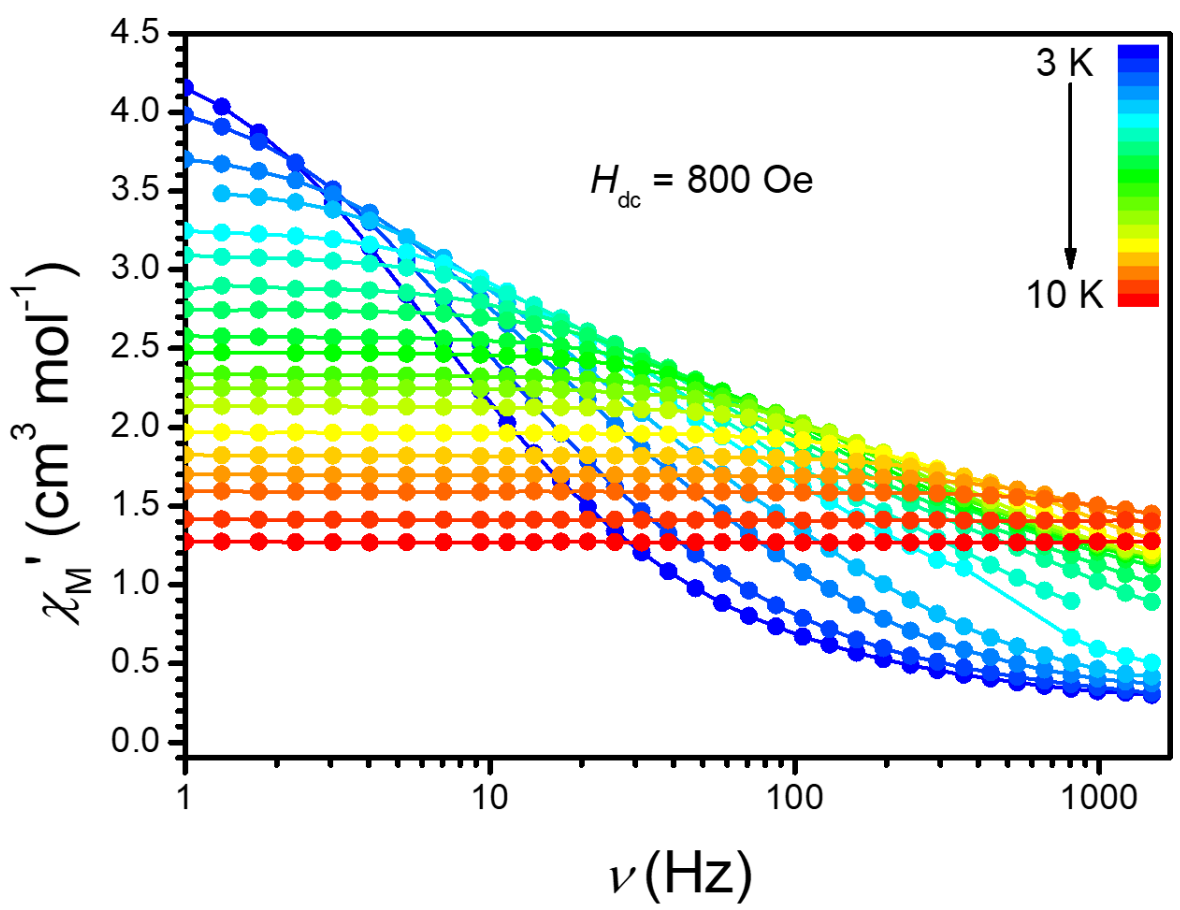

Figure S19. Frequency dependences of $\chi_{\mathrm{M}^{\prime}}$ measured between 3 and $10 \mathrm{~K}$ for $\boldsymbol{R}-\mathbf{1}$ under an 800 Oe field. The solid lines are guides to the eyes. 


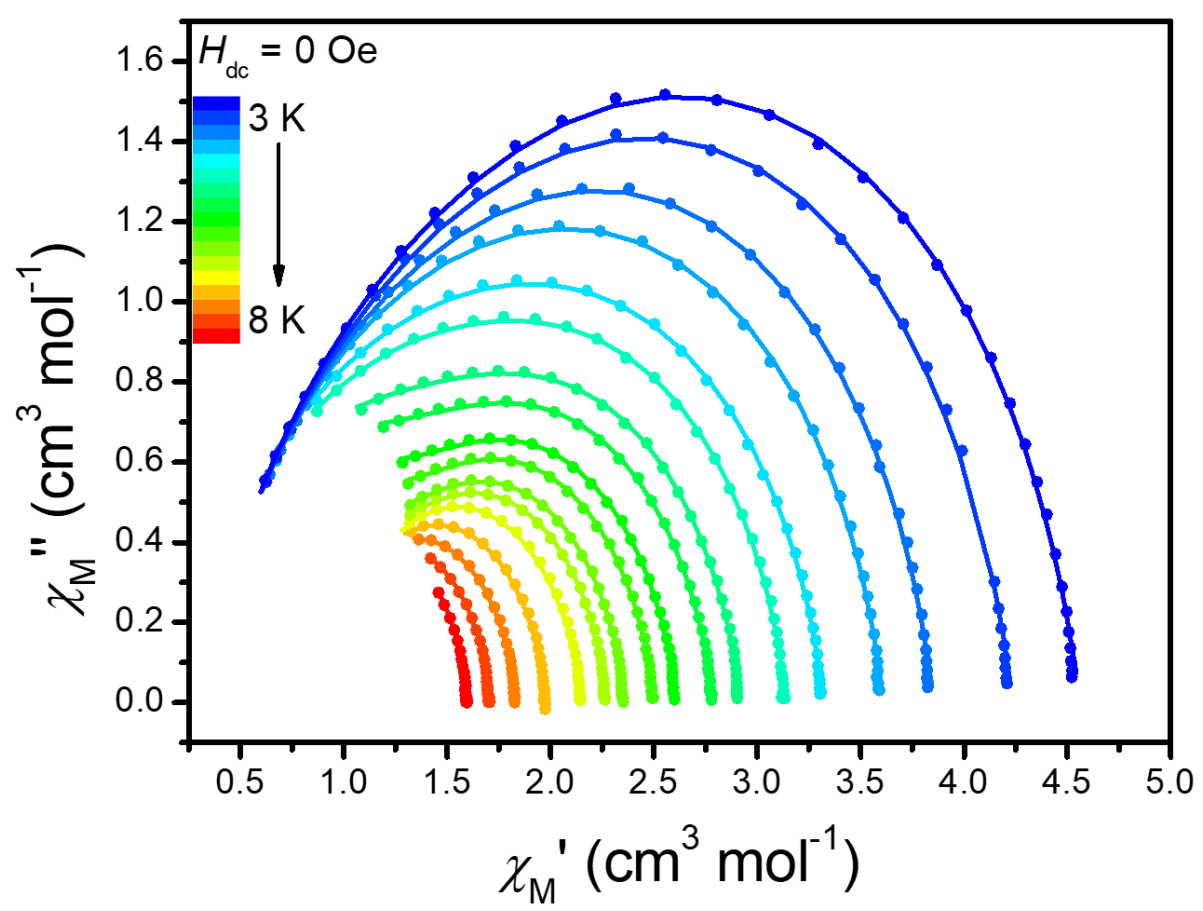

Figure S20. Cole-Cole plots for the ac susceptibilities at 0 Oe for $\boldsymbol{R}-\mathbf{1}$. The solid lines are the best fit with an extended Debye model featuring two relaxation times (solid lines).

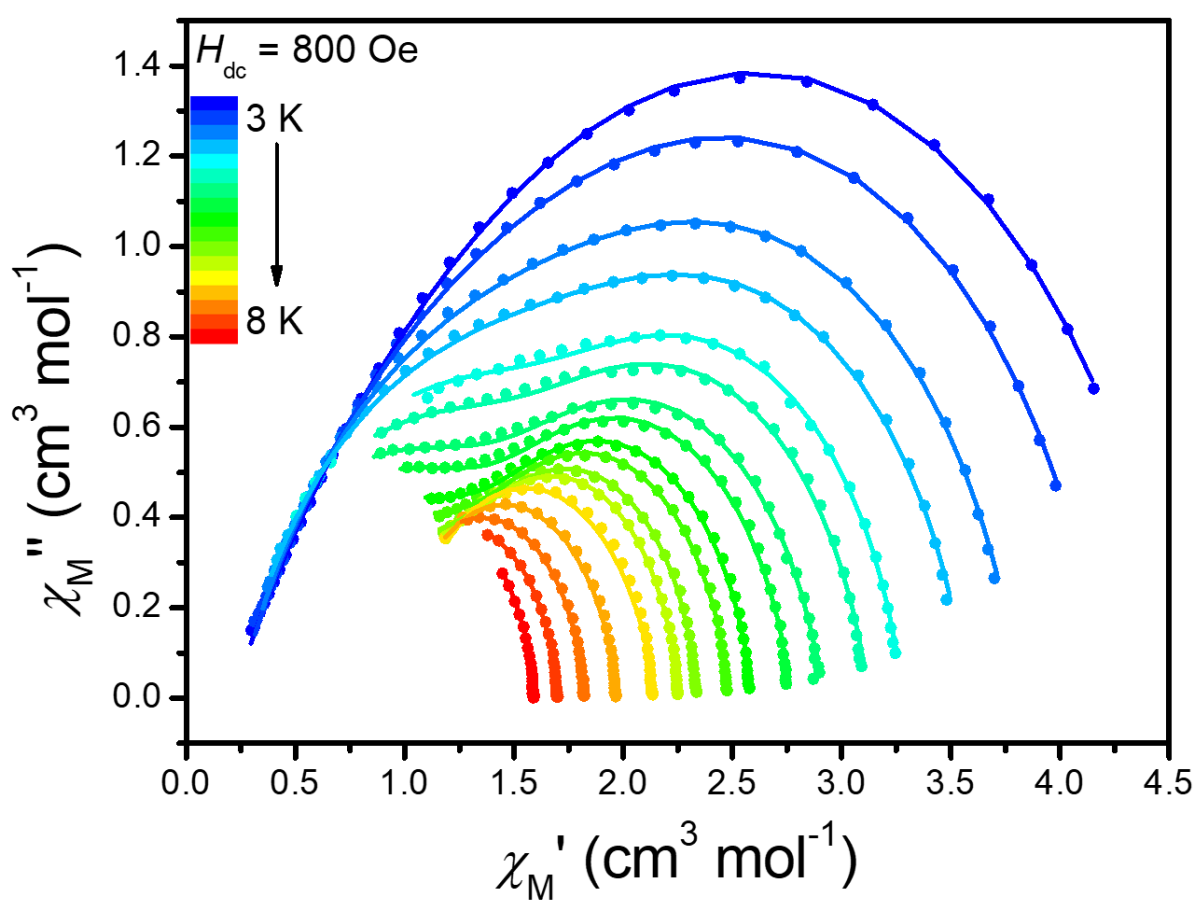

Figure S21. Cole-Cole plots for the ac susceptibilities at 800 Oe for $\boldsymbol{R}-\mathbf{1}$. The solid lines are the best fit with an extended Debye model featuring two relaxation times (solid lines). 


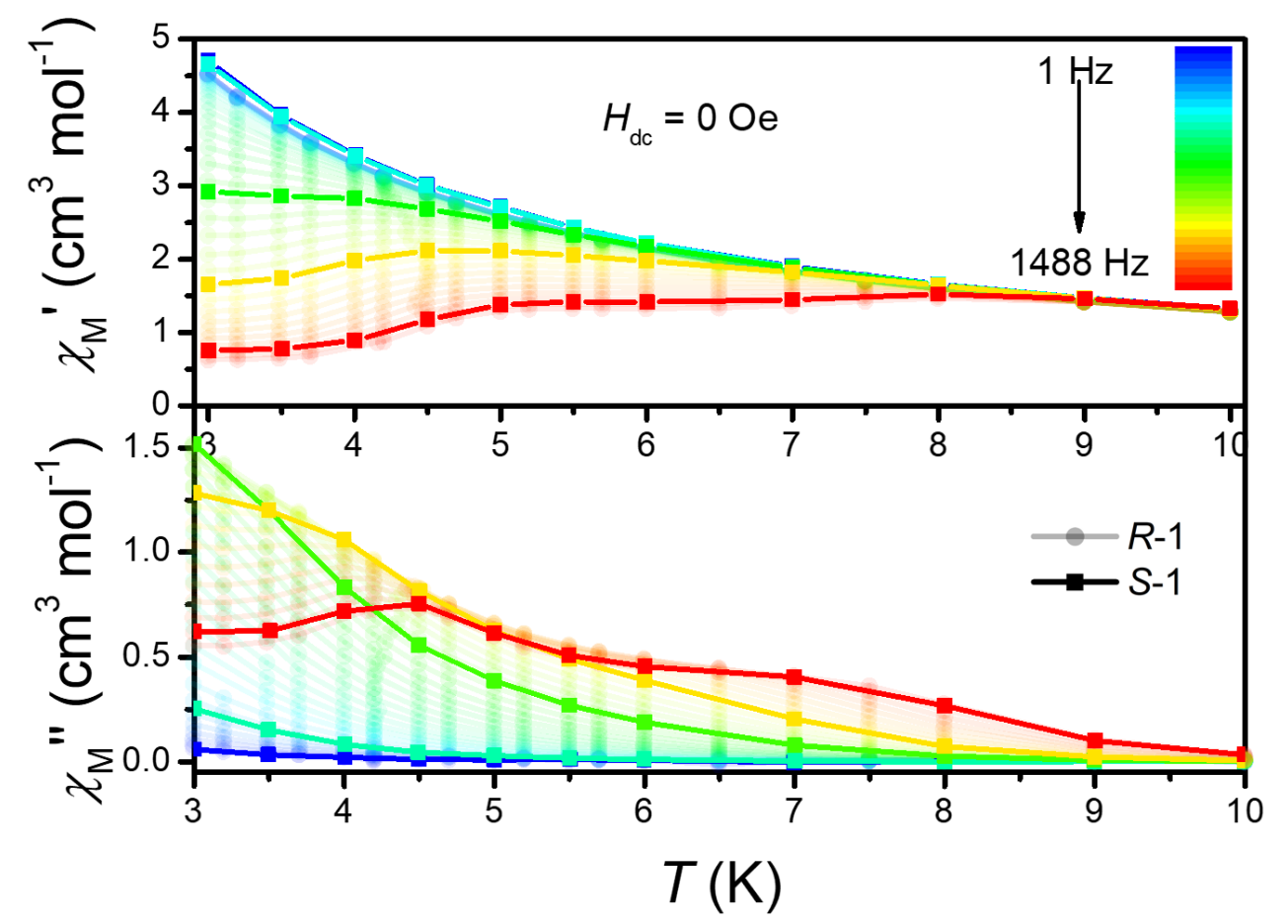

Figure S22. Temperature dependences of $\chi_{\mathrm{M}}{ }^{\prime}$ and $\chi_{\mathrm{M}}{ }^{\prime \prime}$ for $\boldsymbol{R}-\mathbf{1}$ and $\boldsymbol{S}-\mathbf{1}$ under a zero field.

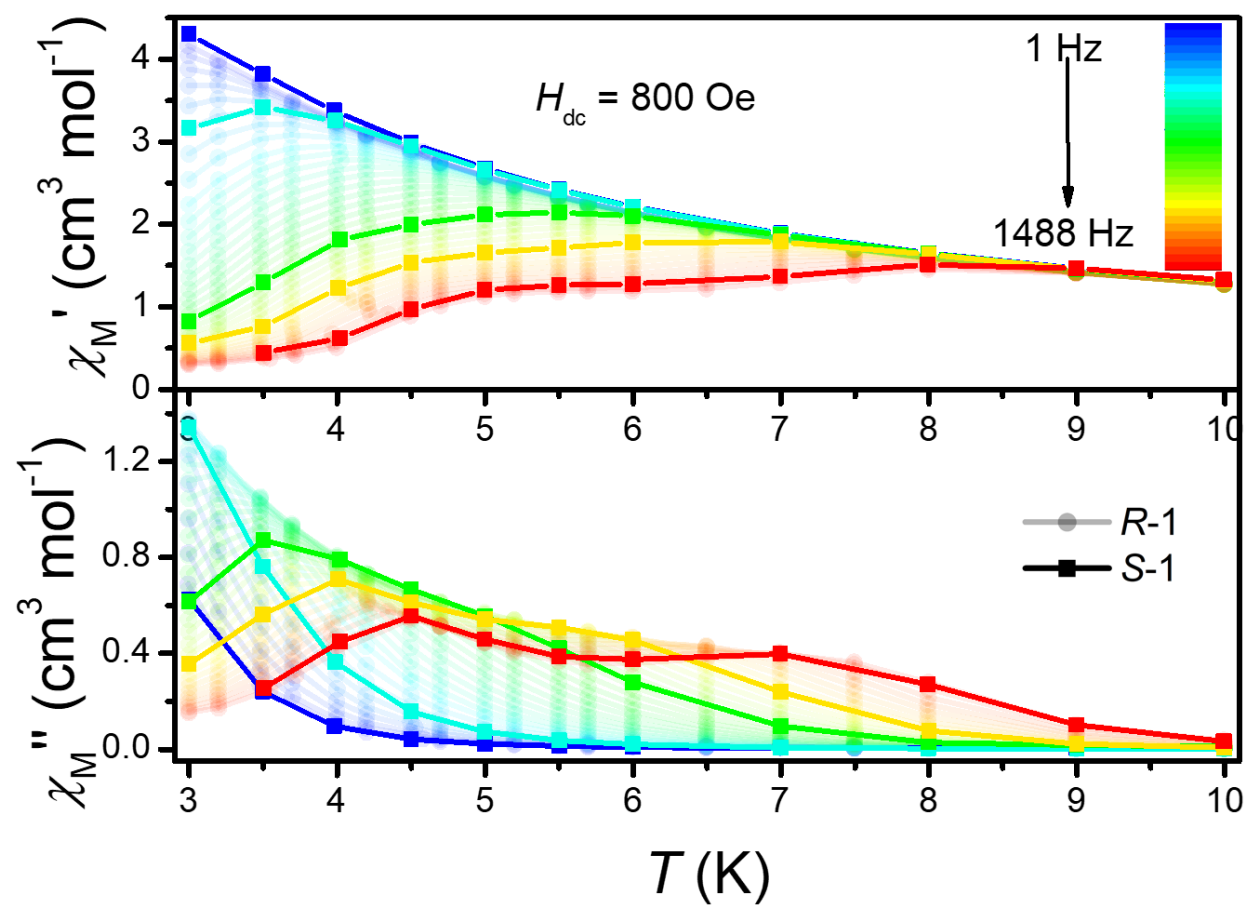

Figure S23. Temperature dependences of $\chi_{\mathrm{M}^{\prime}}$ and $\chi_{\mathrm{M}}{ }^{\prime \prime}$ for $\boldsymbol{R}-\mathbf{1}$ and $\boldsymbol{S}-\mathbf{1}$ under an 800 Oe field. 


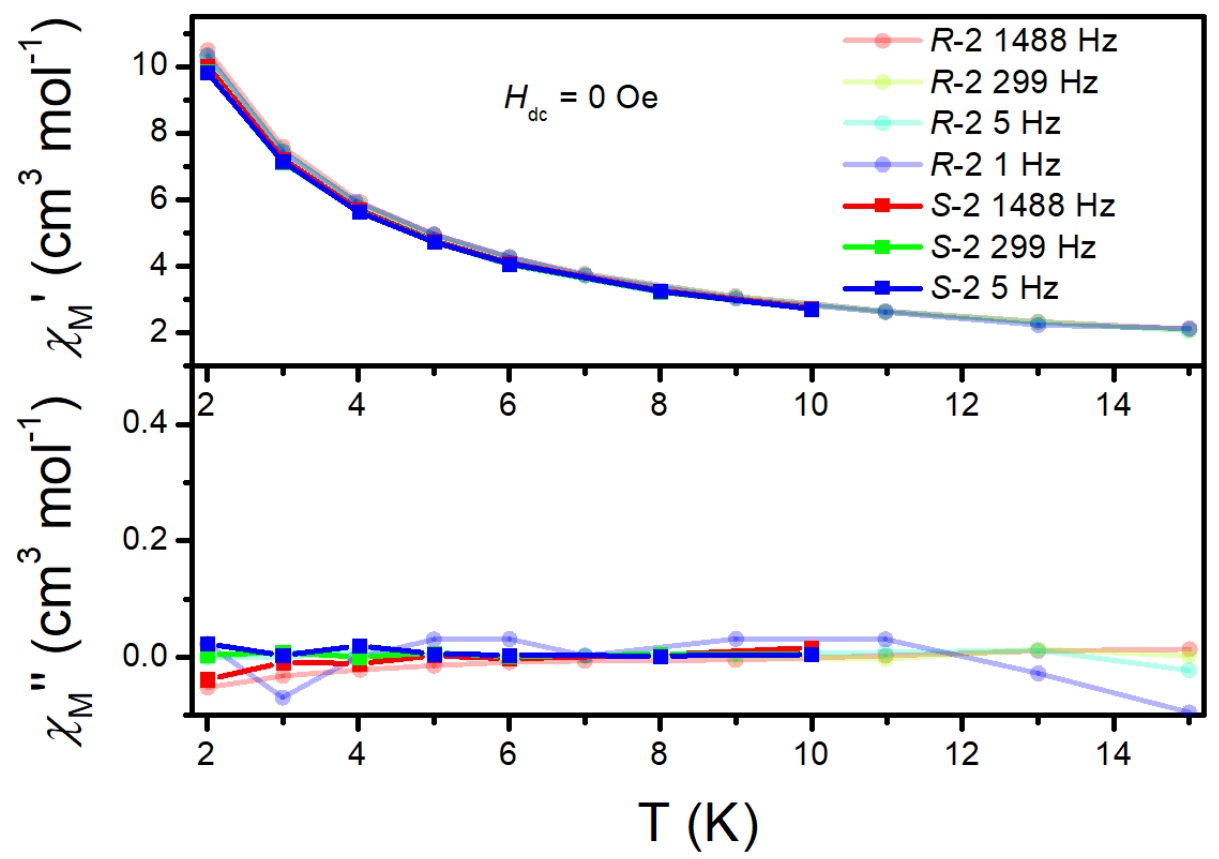

Figure S24. Temperature dependences of $\chi_{\mathrm{M}}{ }^{\prime}$ and $\chi_{\mathrm{M}} \mathrm{M}^{\prime \prime}$ for $\boldsymbol{R}-\mathbf{2}$ and $\boldsymbol{S}-\mathbf{2}$ under a zero field.

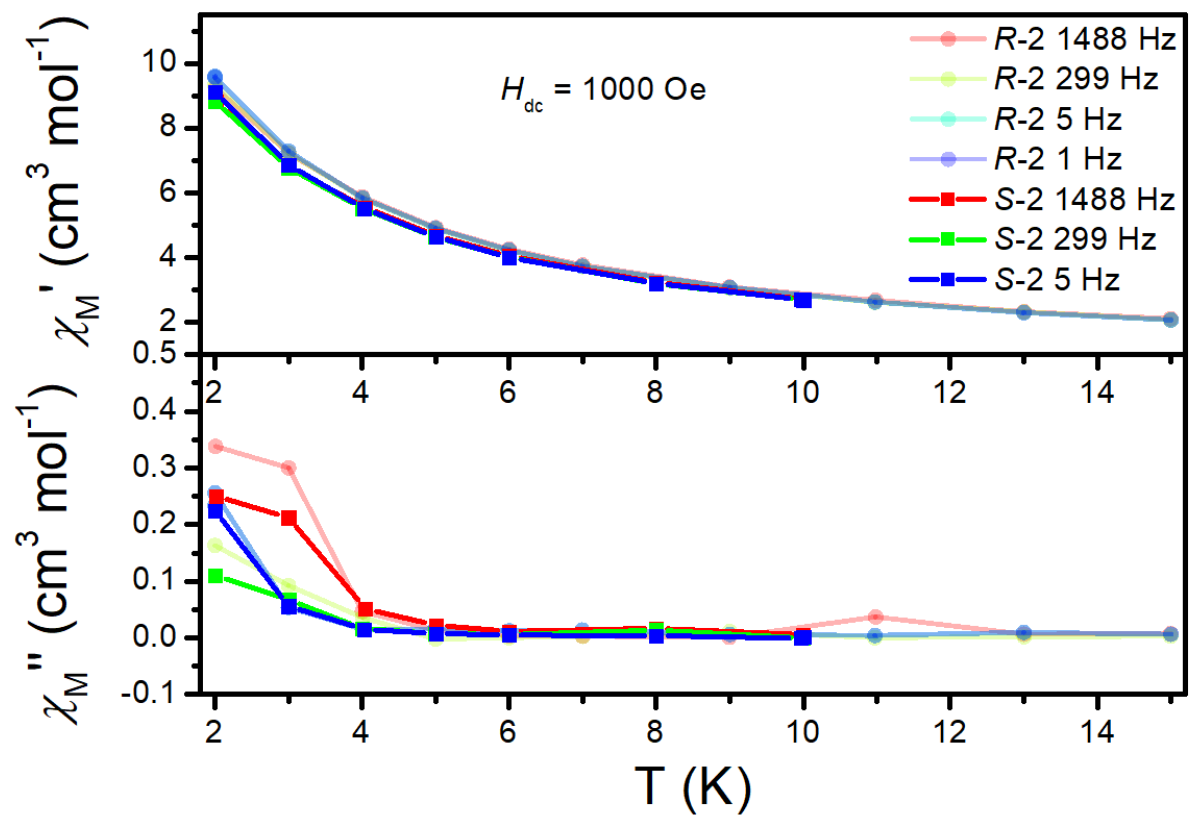

Figure S25. Temperature dependences of $\chi_{\mathrm{M}^{\prime}}$ and $\chi_{\mathrm{M}}{ }^{\prime \prime}$ for $\boldsymbol{R}-\mathbf{2}$ and $\boldsymbol{S}-\mathbf{2}$ under a 1000 Oe field. 


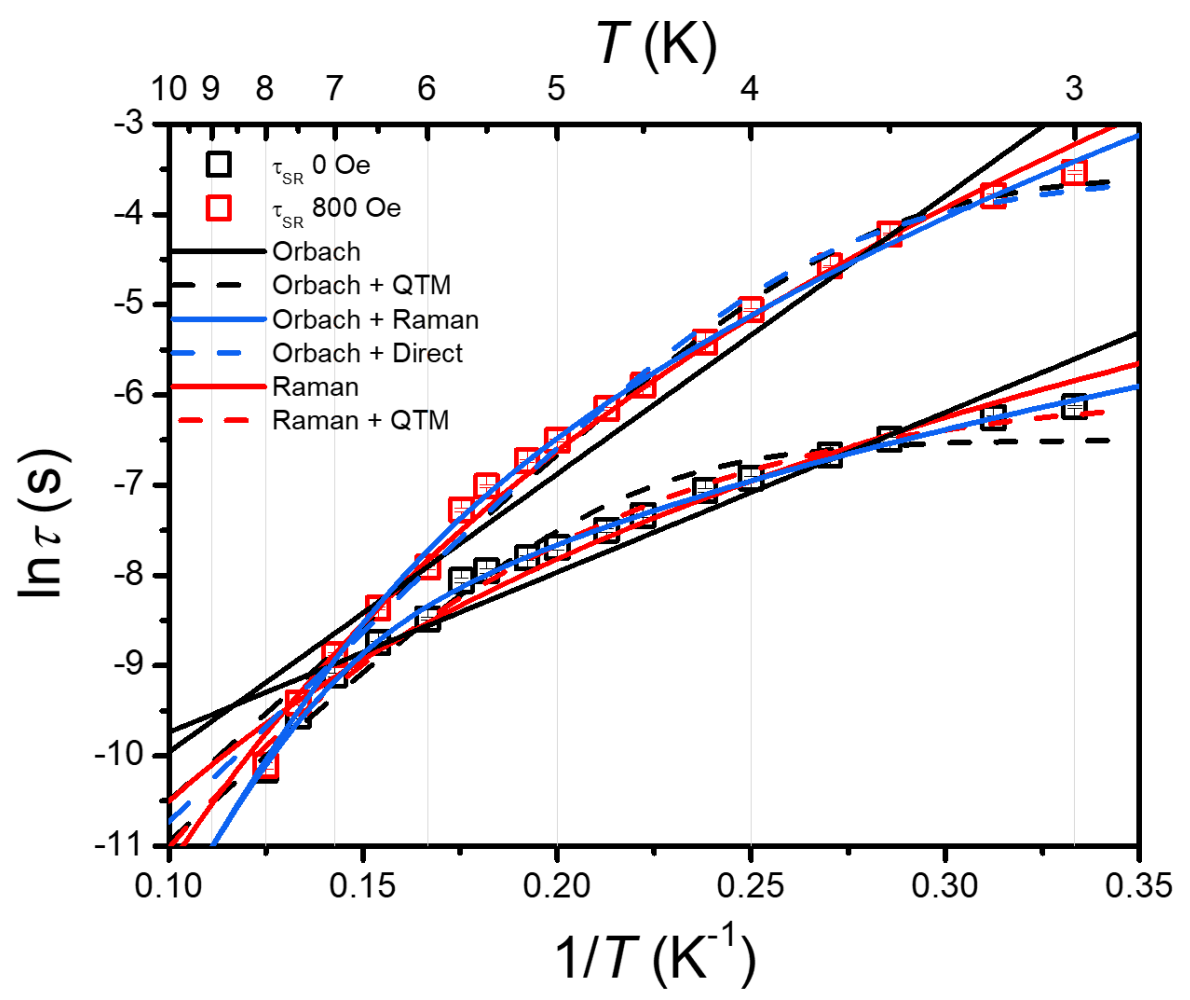

Figure S26. Temperature dependence of the relaxation time $\tau_{\mathrm{SR}}$ under zero (black) and 800 Oe dc magnetic field (red) for $\boldsymbol{R}-1$ in the temperature range of 3-8 K. The lines are the fittings with different relaxation processes.

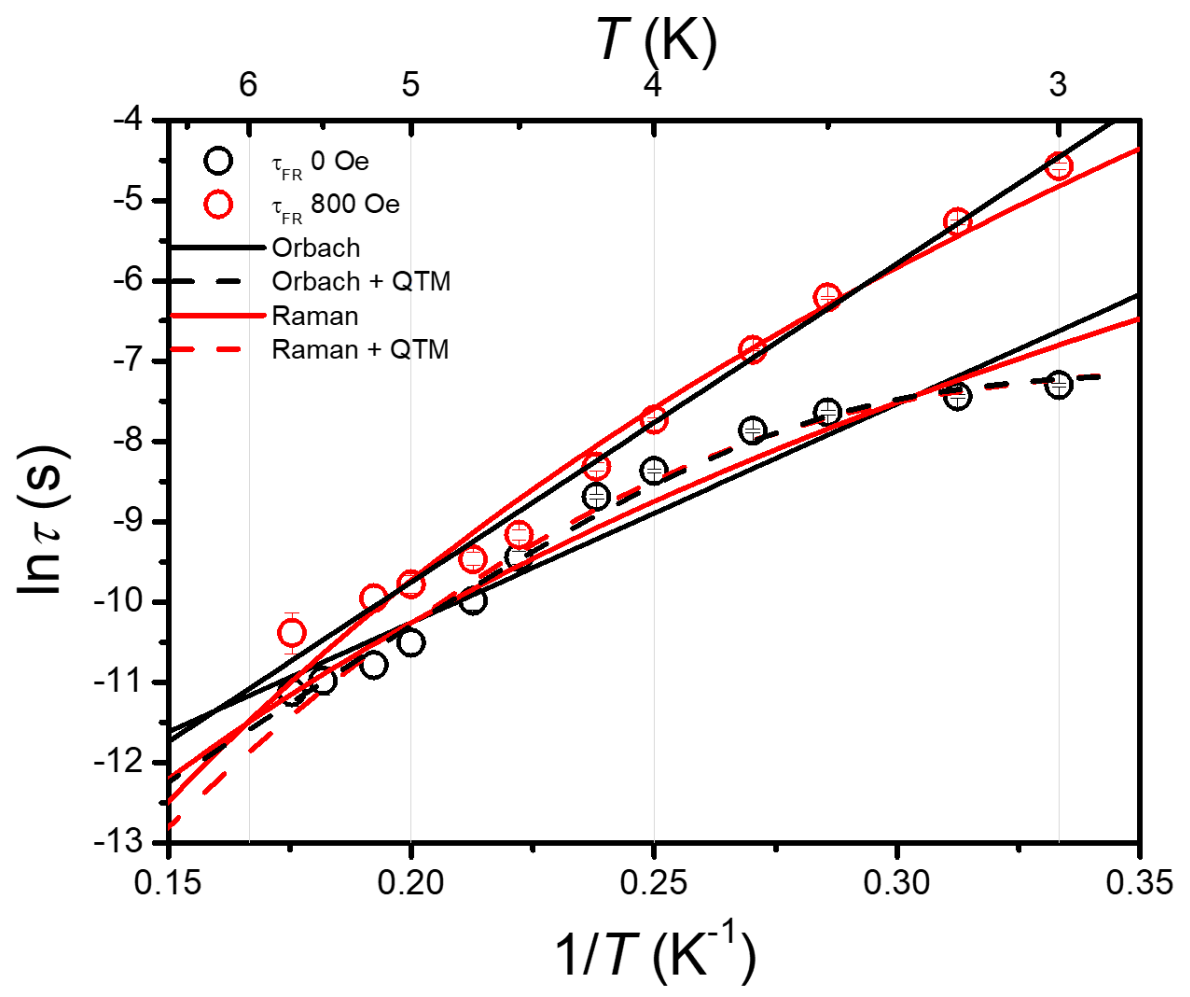

Figure S27. Temperature dependence of the relaxation time $\tau_{\mathrm{FR}}$ under zero (black) and $800 \mathrm{Oe} \mathrm{dc}$ magnetic field (red) for $\boldsymbol{R}-1$ in the temperature range of 3-5.7 K. The lines are the fittings with different relaxation processes. 
Table S11. The relaxation time fitting parameters of with different relaxation processes

\begin{tabular}{|c|c|c|c|c|c|c|c|c|c|c|}
\hline \multirow[b]{2}{*}{ Relaxation } & \multirow[b]{2}{*}{$\begin{array}{c}\mathrm{H} \\
(\mathrm{Oe})\end{array}$} & \multirow[b]{2}{*}{ Fitting processes } & \multicolumn{2}{|c|}{ Orbach } & \multicolumn{2}{|c|}{ Raman } & \multirow{2}{*}{$\begin{array}{c}\text { Direct } \\
\qquad A\end{array}$} & \multirow{2}{*}{$\begin{array}{c}\mathrm{QTM} \\
\tau_{\mathrm{QTM}}(\mathrm{S})\end{array}$} & \multicolumn{2}{|c|}{ Statistics } \\
\hline & & & $\tau_{0}(\mathrm{~s})$ & $U_{\text {eff }}(\mathrm{K})$ & $\begin{array}{c}C \\
\left(\mathrm{~s}^{-1} \mathrm{~K}^{-\mathrm{n}}\right)\end{array}$ & $n$ & & & $\begin{array}{l}\text { Reduced } \\
\text { Chi-Sqr }\end{array}$ & $\begin{array}{l}\text { Adj. R- } \\
\text { Square }\end{array}$ \\
\hline \multirow{6}{*}{ FR } & \multirow{4}{*}{0} & Orbach & $1.5(8) \times 10^{-7}$ & $27(2)$ & & & & & 0.16841 & 0.92163 \\
\hline & & Raman & & & $0.5(3)$ & $6.8(4)$ & & & 0.09189 & 0.95724 \\
\hline & & Orbach + QTM & $1.2(3) \times 10^{-8}$ & $40(1)$ & & & & $8(1) \times 10^{-4}$ & 0.03089 & 0.99057 \\
\hline & & Raman + QTM & & & $0.002(1)$ & $10.1(5)$ & & $7.4(3) \times 10^{-4}$ & 2.99294 & 0.99297 \\
\hline & \multirow{2}{*}{800} & Orbach & $2.1(5) \times 10^{-8}$ & $40(1)$ & & & & & 0.03089 & 0.99057 \\
\hline & & Raman & & & $0.003(2)$ & $9.6(5)$ & & & 0.09863 & 0.97539 \\
\hline \multirow{10}{*}{ SR } & \multirow{5}{*}{0} & Orbach & $1.0(3) \times 10^{-5}$ & $18(1)$ & & & & & 0.12593 & 0.9066 \\
\hline & & Raman & & & $5(1)$ & $3.9(2)$ & & & 0.0411 & 0.96951 \\
\hline & & Orbach + QTM & $4(1) \times 10^{-7}$ & $39(2)$ & & & & $1.5(2) \times 10^{-3}$ & 0.04837 & 0.98354 \\
\hline & & Orbach + Raman & $2(3) \times 10^{-9}$ & 82(9) & $14(2)$ & $3.1(1)$ & & & 0.00618 & 0.9979 \\
\hline & & Raman + QTM & & & $0.5(2)$ & $5.1(3)$ & & $2.7(4) \times 10^{-3}$ & 0.014 & 0.98962 \\
\hline & \multirow{5}{*}{800} & Orbach & $2.2(8) \times 10^{-6}$ & $31(2)$ & & & & & 0.18965 & 0.9513 \\
\hline & & Raman & & & $0.017(4)$ & $6.7(1)$ & & & 0.02805 & 0.9928 \\
\hline & & Orbach + QTM & $6(2) \times 10^{-7}$ & $39(2)$ & & & & $2.9(5) \times 10^{-2}$ & 0.04837 & 0.98354 \\
\hline & & Orbach + Direct & $3(1) \times 10^{-7}$ & $42(2)$ & & & $13(2)$ & & 0.04136 & 0.98938 \\
\hline & & Orbach + Raman & $3(3) \times 10^{-9}$ & 82(9) & $0.04(1)$ & $5.9(2)$ & & & 0.00618 & 0.9979 \\
\hline
\end{tabular}

The fittings in green are considered as reasonable relaxation processes for each curve. Others are excluded for their low Adj. R-Square values or the uncommon $n$ value (see the manuscript). When fitting with an Orbach process, the same relaxation data (i.e. FR or SR) with and without field were fitted simultaneously with a shared $U_{\text {eff }}$ value. 
Table S12. Ab Initio Calculated Energies, g-Tensors, and Wavefunction composition for the Kramers Doublets (KD) of the ground-state multiplet for Er1 and Er2 of $\boldsymbol{R}-\mathbf{1}$.

\begin{tabular}{|c|c|c|c|c|c|}
\hline KD & $\mathrm{E}\left(\mathrm{cm}^{-1}\right)$ & $g_{X}$ & $g_{\mathrm{Y}}$ & $g_{\mathrm{Z}}$ & The percentage of wavefunction composition* \\
\hline \multicolumn{6}{|c|}{ Er1 } \\
\hline 1 & 0 & 0.0676 & 0.1438 & 16.9905 & $0.82| \pm 15 / 2>+0.12| \pm 11 / 2\rangle$ \\
\hline 2 & 70.337 & 2.2550 & 5.6878 & 10.5443 & $\begin{array}{l}0.28| \pm 13 / 2>+0.18| \pm 9 / 2>+0.14 \mid \pm 5 / 2>+0.12 \\
| \pm 11 / 2>+0.12| \pm 3 / 2>\end{array}$ \\
\hline 3 & 101.530 & 1.6624 & 3.2357 & 8.2481 & $0.24| \pm 7 / 2>+0.18| \pm 5 / 2>+0.17| \pm 1 / 2>+0.13| \pm 13 / 2>$ \\
\hline 4 & 137.627 & 1.2019 & 1.9130 & 12.5630 & $\begin{array}{l}0.28| \pm 3 / 2>+0.20| \pm 5 / 2>+0.17| \pm 7 / 2>+0.12| \pm 1 / 2> \\
+0.11 \mid \pm 9 / 2>\end{array}$ \\
\hline 5 & 213.034 & 0.0602 & 0.9011 & 12.6158 & $0.51| \pm 1 / 2>+0.14| \pm 3 / 2>+0.11 \mid \pm 7 / 2>$ \\
\hline 6 & 239.003 & 1.0409 & 1.9519 & 11.0642 & $\begin{array}{l}0.22| \pm 5 / 2>+0.21| \pm 9 / 2>+0.18 \mid \pm 13 / 2>+0.14 \\
| \pm 3 / 2>+0.13| \pm 7 / 2>\end{array}$ \\
\hline 7 & 274.938 & 0.6260 & 2.8489 & 14.1658 & $0.23| \pm 3 / 2>+0.21| \pm 5 / 2>+0.19| \pm 7 / 2>+0.14| \pm 1 / 2>$ \\
\hline 8 & 314.332 & 0.1059 & 0.1880 & 17.2296 & $\begin{array}{l}0.40| \pm 11 / 2>+0.21| \pm 13 / 2>+0.20 \mid \pm 9 / 2>+0.12 \\
\mid \pm 15 / 2>\end{array}$ \\
\hline \multicolumn{6}{|c|}{ Er2 } \\
\hline 1 & 0 & 0.1397 & 0.2515 & 16.4224 & $0.71| \pm 15 / 2>+0.20| \pm 11 / 2>$ \\
\hline 2 & 51.044 & 1.1901 & 2.0870 & 12.4963 & $0.36| \pm 9 / 2>+0.21| \pm 13 / 2>+0.20 \mid \pm 5 / 2>$ \\
\hline 3 & 90.281 & 3.7479 & 4.7045 & 8.2924 & $0.45| \pm 7 / 2>+0.11| \pm 5 / 2>$ \\
\hline 4 & 113.898 & 1.2413 & 2.4116 & 12.7340 & $0.32| \pm 3 / 2>+0.29| \pm 5 / 2>+0.16 \mid \pm 7 / 2>$ \\
\hline 5 & 216.172 & 0.2869 & 0.7621 & 11.9361 & $0.59 \mid \pm 1 / 2>$ \\
\hline 6 & 256.283 & 1.9040 & 4.8576 & 9.2556 & $0.25| \pm 3 / 2>+0.18| \pm 13 / 2>+0.16| \pm 5 / 2\rangle$ \\
\hline 7 & 294.539 & 1.3020 & 4.7654 & 12.3480 & $\begin{array}{l}0.18| \pm 1 / 2>+0.18| \pm 3 / 2\rangle+0.14 \mid \pm 5 / 2>+0.14 \\
| \pm 13 / 2>+0.13| \pm 9 / 2>+0.12| \pm 7 / 2>+0.11| \pm 11 / 2\rangle\end{array}$ \\
\hline 8 & 334.284 & 0.0770 & 0.3170 & 16.7727 & $0.34| \pm 11 / 2>+0.32| \pm 13 / 2>+0.20 \mid \pm 15 / 2>$ \\
\hline
\end{tabular}

*Only the contributions $\geq 10 \%$ are given. 


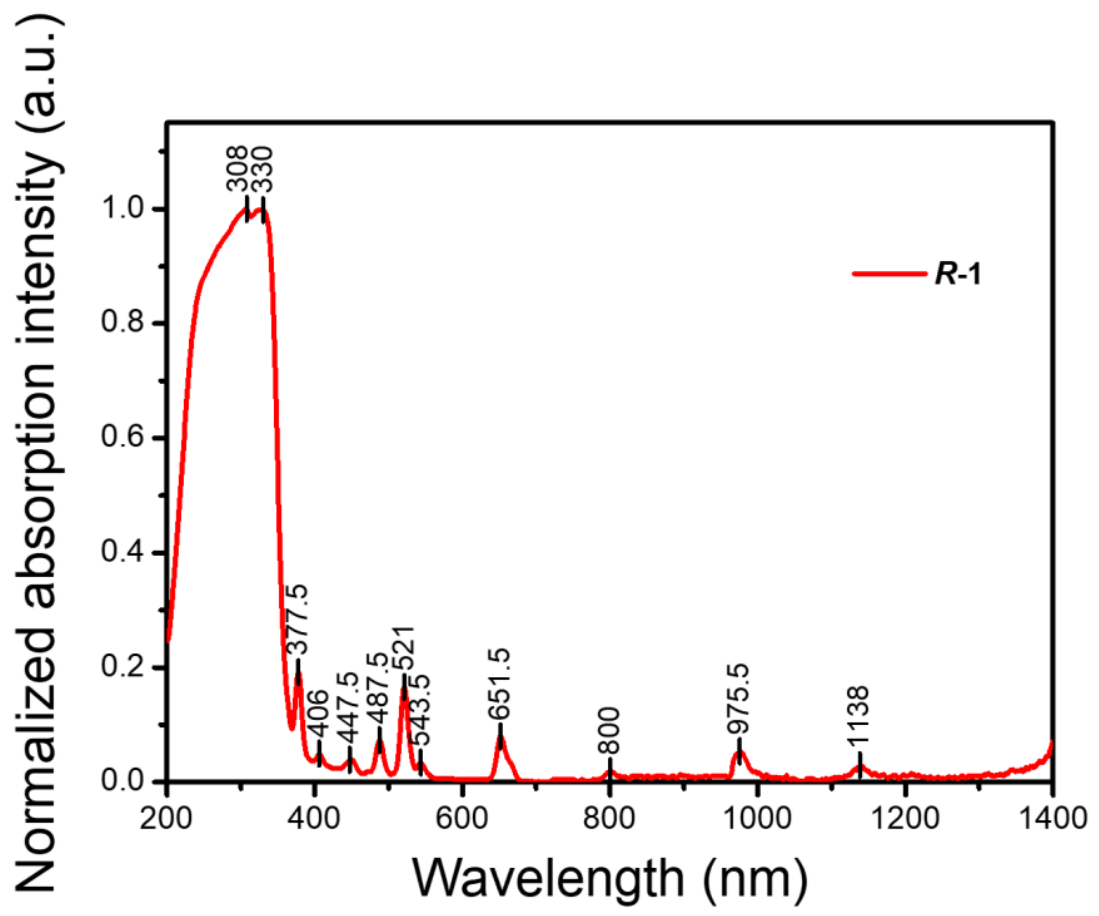

Figure S28. Normalized UV-vis-NIR absorption spectrum of $\boldsymbol{R}-\mathbf{1}$ in solid state.

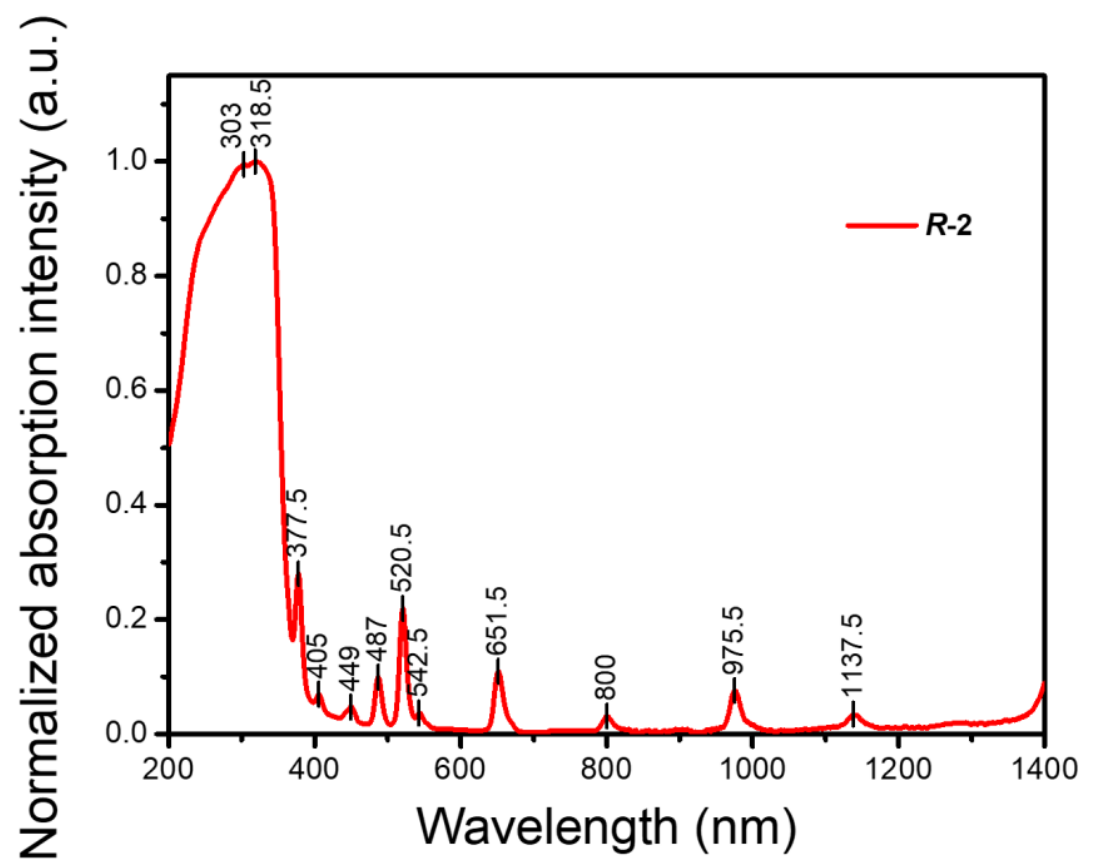

Figure S29. Normalized UV-vis-NIR absorption spectrum of $\boldsymbol{R}-\mathbf{2}$ in solid state.

\section{References:}

1. Llunell, M.; Casanova, D.; Cirera, J.; Bofill, J. M.; Alemany, P.; Alvarez, S. SHAPE (v. 2.1), Barcelona, 2013.

2. Alvarez, S.; Alemany, P.; Avnir, D. Continuous chirality measures in transition metal chemistry. Chem. Soc. Rev. 2005, 34 (4), 313-326.

3. Fujii, I.; Hirayama, N. Helv. Chim. Acta 2002, 85, 2946-2960. 